\title{
SPECTRAL FACTORIZATION OF 2-BLOCK TOEPLITZ MATRICES AND REFINEMENT EQUATIONS
}

\author{
V. YU. PROTASOV
}

\begin{abstract}
Pairs of 2-block Toeplitz $(N \times N)$-matrices $\left(T_{s}\right)_{i j}=p_{2 i-j+s-1}, s=0,1$, $i, j \in\{1, \ldots, N\}$, are considered for arbitrary sequences of complex coefficients $p_{0}, \ldots, p_{N}$. A complete spectral resolution of the matrices $T_{0}, T_{1}$ in the system of their common invariant subspaces is obtained. A criterion of nondegeneracy and of irreducibility of these matrices is derived, and their kernels, root subspaces, and all common invariant subspaces are found explicitly. The results are applied to the study of refinement functional equations and also subdivision and cascade approximation algorithms. In particular, the well-known formula for the exponent of regularity of a refinable function is simplified. A factorization theorem that represents solutions of refinement equations by certain convolutions is obtained, along with a characterization of the manifold of smooth refinable functions. The problem of continuity of solutions of the refinement equations with respect to their coefficients is solved. A criterion of convergence of the corresponding cascade algorithms is obtained, and the rate of convergence is computed.
\end{abstract}

\section{§1. INTRODUCTION}

The finite-dimensional 2-Toeplitz operators corresponding to a given sequence of complex numbers $p_{0}, \ldots, p_{N}$ are the operators $T_{0}, T_{1}$ acting in $\mathbb{R}^{N}$ and determined by the following matrices:

$$
\left(T_{0}\right)_{i j}=p_{2 i-j-1}, \quad\left(T_{1}\right)_{i j}=p_{2 i-j}, \quad i, j \in\{1, \ldots, N\}
$$

(we set $p_{k}=0$ for $k<0$ and for $k>N$ ). Usually, the matrices $T_{0}, T_{1}$ are referred to in the literature as 2-block, or $(2 \times 1)$-block, or two-slanted Toeplitz matrices. For simplicity, in the sequel we call them simply Toeplitz matrices, although this differs from the conventional definition. The linear operators corresponding to the matrices (1) will be denoted by the same symbols and will also be called Toeplitz operators. Without loss of generality, at the expense of a possible shift of the argument and a change of the dimension, it may be assumed that $p_{0} p_{N} \neq 0$. For example, if $N=6$, then the Toeplitz matrices are

$$
T_{0}=\left(\begin{array}{cccccc}
p_{0} & 0 & 0 & 0 & 0 & 0 \\
p_{2} & p_{1} & p_{0} & 0 & 0 & 0 \\
p_{4} & p_{3} & p_{2} & p_{1} & p_{0} & 0 \\
p_{6} & p_{5} & p_{4} & p_{3} & p_{2} & p_{1} \\
0 & 0 & p_{6} & p_{5} & p_{4} & p_{3} \\
0 & 0 & 0 & 0 & p_{6} & p_{5}
\end{array}\right), \quad T_{1}=\left(\begin{array}{cccccc}
p_{1} & p_{0} & 0 & 0 & 0 & 0 \\
p_{3} & p_{2} & p_{1} & p_{0} & 0 & 0 \\
p_{5} & p_{4} & p_{3} & p_{2} & p_{1} & p_{0} \\
0 & p_{6} & p_{5} & p_{4} & p_{3} & p_{2} \\
0 & 0 & 0 & p_{6} & p_{5} & p_{4} \\
0 & 0 & 0 & 0 & 0 & p_{6}
\end{array}\right) .
$$

2000 Mathematics Subject Classification. Primary 39B22, 15A23, 26C10, 26 A30.

Key words and phrases. Matrix factorization, spectrum, polynomial, cyclic trees, refinement equation, approximation algorithms, wavelets.

Supported by RFBR (grant no. 05-01-00066) and by Leading Scientific Schools (grant no. $5813.2006 .1)$. 
Various properties of the Toeplitz matrices (1) have been studied in many publications, due to their applications to wavelet theory, refinement equations, approximation theory, fractal curves (subdivision algorithms, de Rham curves, etc.), number theory, and combinatorics (see [1]-13] and the references therein). The main problems are to characterize the spectra of Toeplitz operators, their kernels, root subspaces, common invariant subspaces, and to compute their joint spectral radii. All results of this paper can be naturally extended to the case of $k$-block Toeplitz matrices, where $k$ matrices $\left(T_{s}\right)_{i j}=T_{k(i-1)-(j-1)+s}, s=0,1, \ldots, k-1$, are associated with a sequence $\left\{p_{i}\right\}$. For simplicity, we restrict ourselves to the case where $k=2$.

In Chapter I we consider general Toeplitz operators (11) corresponding to an arbitrary sequence $p_{0}, \ldots, p_{N}$. We prove a criterion for the nondegeneracy of the operators $T_{0}, T_{1}$ (see Proposition 1) and a criterion for their irreducibility (see Proposition 2). The irreducibility property of a pair of operators $T_{0}, T_{1}$ means that they have no common nontrivial invariant subspaces (real or complex). Those criteria are formulated in terms of roots of the generating function

$$
\mathbf{p}(z)=\sum_{k=0}^{N} p_{k} z^{k}
$$

We show that the operators $T_{0}, T_{1}$ are both nondegenerate if and only if the polynomial $\mathbf{p}$ has no so-called symmetric zeros. Furthermore, if these operators turn out to be degenerate, then the kernels of the adjoint matrices $T_{0}^{*}, T_{1}^{*}$ coincide, and the same is true for their root subspaces of all orders. A pair $T_{0}, T_{1}$ is irreducible if and only if these operators are nondegenerate and $\mathbf{p}$ has no cycles, i.e., there are no finite cyclic (with respect to squaring) sets $\mathbf{b}$ on the unit circle such that $\mathbf{p}(-\mathbf{b})=0$. In $\S 3$ we elaborate upon an algorithm of cleaning that eliminates all symmetric zeros and cycles for an arbitrary polynomial p. It is shown that the resulting "clean" polynomial $\tilde{\mathbf{p}}$ does not depend on the order of removing symmetric zeros and cycles.

This algorithm reduces many problems on Toeplitz operators to the case of clean polynomials, making it possible to reduce the dimension and to exploit some specific properties of operators of clean polynomials (nondegeneracy, irreducibility, etc.). In $\S \S 4$ and 5, all root subspaces and common invariant subspaces are found explicitly for arbitrary Toeplitz operators. The description of the structure of those subspaces involves elementary graph theory (binary trees, cyclic trees, and cut sets). We obtain a complete spectral factorization of Toeplitz operators, which gives their explicit form on each common invariant subspace.

Chapters II, III, and IV are devoted to applications of the results obtained in the study of refinement equations, of cascade algorithms, and of subdivision schemes.

A refinement equation (a difference equation with double contraction of the argument) is an equation of the form

$$
\varphi(x)=\sum_{k=0}^{N} c_{k} \varphi(2 x-k), \quad x \in \mathbb{R},
$$

where $c_{0}, \ldots, c_{N}$ is an arbitrary sequence of complex numbers (the mask) satisfying the conditions $c_{0} c_{N} \neq 0, \sum_{k} c_{k}=2$. The polynomial $\mathbf{m}(z)=\frac{1}{2} \sum_{k=0}^{N} c_{k} z^{k}$ is called a symbol of the equation. Clearly, $\mathbf{m}=\frac{1}{2} \mathbf{p}$, where $\mathbf{p}$ is the generating polynomial (2) of the sequence $\left\{c_{k}\right\}$. A refinable function is a compactly supported solution of this equation in the space $\mathcal{S}^{\prime}$ of tempered distributions. It is well known that any refinement equation has a unique, up to multiplication by a constant, compactly supported solution in the space $\mathcal{S}^{\prime}$. Furthermore, the support of this solution is contained in the segment $[0, N]$, and $\int \varphi \neq 0$, which enables us to consider only normalized solutions. Everywhere 
in the sequel we put $\int \varphi=1$. There is an extensive literature devoted to refinement equations. The interest in this subject is caused mainly by its role in wavelet theory [14, as well as by numerous applications to approximation theory, probability, number theory, mathematical physics, etc.; see [3]-[11].

The most well-known refinable functions are the cardinal $B$-splines $B_{k}(x)$ defined as follows: the $B$-spline $B_{0}(x)=\chi_{[0,1)}$ is the indicator function of the half-interval $[0,1)$, and $B_{k+1}=B_{k} * B_{0}$ for any $k \geq 0$, where $[f * g](x)=\int_{\mathbb{R}} f(x-t) g(t) d t$ is the convolution of two functions. The $B$-spline $B_{k}$ satisfies the refinement equation with symbol $\mathbf{m}(z)=\left(\frac{z+1}{2}\right)^{k+1}$. They are not the only refinable splines. For every $N$ there are finitely many splines that are solutions of refinement equations of order $N$. All of them were classified in [3, 16, 17. Those splines exhaust the set of "good" refinable functions. All others are not piecewise analytic and possess fractal-like properties (varying local regularity, etc.) [17. They are not defined by explicit formulas, but constructed by iterative algorithms. One of the main problems of the theory of refinement equations is to determine the regularity of the solution $\varphi$ by the coefficients $\left\{c_{k}\right\}$. Does the solution $\varphi$ belong to $L_{p}$, is it continuous or differentiable? The refinable functions always have a finite smoothness. Among all refinable functions of order $N$, only the $B$-spline $B_{N-1}$ belongs to the space $C^{N-2}(\mathbb{R})$; see 4 .

In the sequel we use the standard notation $C^{l}$ and $W_{p}^{l}$ for the space of functions with continuous $l$ th derivative and, respectively, for the Sobolev space of functions $f$ with an absolutely continuous derivative $f^{(l-1)}$ and with $f^{(l)} \in L_{p}, l \geq 1, p \in[1,+\infty]$. Also, $W_{p}^{0}=L_{p}(\mathbb{R})$, and $C^{0}=C(\mathbb{R})$ is the space of continuous functions. We denote by $\omega_{p}(f, h)=\sup _{|t|<h}\|f(\cdot+t)-f(\cdot)\|_{p}$ the modulus of continuity in $L_{p}$, where $\|f\|_{p}$ is the $L_{p}$-norm. For a given function $f \in L_{p}, p<\infty$, the Hölder exponent in $L_{p}$ is $\alpha_{\varphi, p}=k+\sup \left\{\alpha|| \omega_{p}\left(\varphi^{(k)}, h\right) \mid \leq C h^{\alpha}\right\}$, where $k$ is the largest integer for which $\varphi \in W_{p}^{k}$. Similarly, the Hölder exponent in $C$ is $\alpha_{\varphi}=\alpha_{\varphi, \infty}=k+\sup \left\{\alpha|| \omega_{\infty}\left(\varphi^{(k)}, h\right) \mid \leq C h^{\alpha}\right\}$, where $k$ is the largest integer for which $\varphi \in C^{k}$.

The main method of computing the regularity of refinable functions was elaborated upon in [3, 4, 18. It involves the Toeplitz operators (11) corresponding to the coefficients of the equation $p_{k}=c_{k}$. In [4, 5, 19] it was proved that the regularity exponent $\alpha_{\varphi, p}$ is expressed in terms of the $p$-radius $\rho_{p}$ of the operators $T_{0}, T_{1}$ restricted to a certain subspace $V \subset \mathbb{R}^{N}$. Moreover, $\varphi \in L_{p}$ if and only if $\rho_{p}<1$, and $\varphi \in C$ if and only if $\rho_{\infty}<1$. The $p$-radius (the joint spectral radius, $p \in[1,+\infty]$ ) of two linear operators $A_{0}, A_{1}$ is the quantity

$$
\rho_{p}\left(A_{0}, A_{1}\right)=\lim _{k \rightarrow \infty}\left(2^{-k} \sum_{d_{1}, \ldots, d_{k} \in\{0,1\}}\left\|A_{d_{1}} \cdots A_{d_{k}}\right\|^{p}\right)^{1 / k p}
$$

(for $p=\infty$ we have $\rho_{\infty}=\lim _{k \rightarrow \infty} \max _{d_{1}, \ldots, d_{k} \in\{0,1\}}\left\|A_{d_{1}} \cdots A_{d_{k}}\right\|^{1 / k}$ ). There are several algorithms of estimation of $\rho_{p}$ (see [20-23]). However, the problem remains how to find the subspace $V$ and the restrictions of the operators $T_{0}, T_{1}$ to that subspace. This problem was studied in detail in $3,5,9,10$. In $\S 8$ we obtain a complete solution, which will allow us to simplify the well-known formulas for the regularity exponents of refinable functions and to obtain sharp bounds for their moduli of continuity (see Theorems 9 and 10 in $\S 9$ ). In Chapter IV we characterize the manifold of smooth refinable functions and describe an algorithm of numerical computation of solutions for arbitrary refinement equations. In the case of small degrees of the clean polynomial $\tilde{\mathbf{m}}$, the regularity exponents are found explicitly (see $\S 16$ ). The next problem is to analyze the continuity of solutions of equation (3) with respect to its coefficients $\left\{c_{k}\right\}$. The refinement equations are well known to be unstable: small perturbations of coefficients may lead to jumps of the solution $\varphi$ 
(see [4, 5]). A criterion for continuous dependence on the coefficients is presented in $\S 14$ (Theorem 14).

In Chapter III, the results on the spectral properties of Toeplitz operators are applied to the analysis of convergence of subdivision schemes and cascade algorithms (approximation theory and wavelet theory). In particular, we obtain a sharp criterion of convergence in the spaces $C^{l}$ and $W_{p}^{l}$ (Theorem 11) and compute the rate of convergence (Theorem 13). In $\S 11$ we use these results to analyze some special cases important in applications (interpolatory schemes, schemes with nonnegative coefficients, etc.).

\section{Chapter I. Spectral Resolution of Toeplitz matrices}

\section{§2. Irreducible Toeplitz operators}

For an arbitrary sequence $\left\{p_{k}\right\}_{k=0}^{N}$ with $p_{0} p_{N} \neq 0$, we consider a pair of Toeplitz operators (11). First, we are going to deduce conditions for the nondegeneracy of these operators and for their irreducibility, i.e., the absence of common nontrivial invariant subspaces. These conditions are formulated in terms of zeros of the generating polynomial (2). A complex number $a \in \mathbb{C} \backslash\{0\}$ is called a symmetric zero of the polynomial $\mathbf{p}$ if $\mathbf{p}(\sqrt{a})=\mathbf{p}(-\sqrt{a})=0$. In this case, the pair of numbers $\pm \sqrt{a}$ is called a pair of symmetric zeros for $\mathbf{p}$. A finite set $\mathbf{b}=\left\{b_{1}, \ldots, b_{n}\right\}$ of distinct complex numbers is cyclic if $b_{1}^{2}=b_{2}, b_{2}^{2}=b_{3}, \ldots, b_{n-1}^{2}=b_{n}, b_{n}^{2}=b_{1}$. For any $n$ there exist finitely many cyclic sets of order $n$, all of them lying on the unit circle. Furthermore, $b_{1}=e^{\frac{2 \pi r i}{2^{n}-1}}$, where the natural number $r \leq 2^{n}-1$ is such that for every $k<n$ the number $r\left(2^{k}-1\right)$ is not divisible by $2^{n}-1$. The simplest cyclic set $\mathbf{b}=\{1\}$ is said to be trivial; all others are nontrivial. A cycle of a polynomial $\mathbf{p}$ is a cyclic set $\mathbf{b}$ such that $\mathbf{p}(-\mathbf{b})=0$. We say that a polynomial $\mathbf{p}$ is clean if it has neither symmetric zeros nor cycles.

Theorem 1. If a polynomial $\mathbf{p}$ is clean, then the operators $T_{0}, T_{1}$ are both nondegenerate and irreducible.

The converse is also true even in a stronger sense. Propositions 1 and 2 provide criteria for nondegeneracy and for irreducibility. In its turn, Theorem 1 is a direct consequence of those propositions.

For a nonzero complex number $z$, the vector $\left(1, z, \ldots, z^{N-1}\right)^{T} \in \mathbb{R}^{N}$ will be denoted by $[z]$. Thus, $[z]_{k}=z^{k-1}$. For $l \geq 1$, we denote by $[z]^{l}$ the vector in $\mathbb{R}^{N}$ with the coordinates $[z]_{k}^{l}=(k-1)^{l} z^{k-1}$. Also, we set $[z]^{0}=[z]$. For distinct complex numbers $z_{1}, \ldots, z_{q}$ and for positive integers $r_{1}, \ldots, r_{q}$ such that $m=\sum_{j} r_{j} \leq N$, the $m$ vectors $\left\{\left[z_{j}\right]^{s}, s=0, \ldots, r_{j}-1, j=1, \ldots, q\right\}$ are linearly independent [15].

Proposition 1. If $\mathbf{p}$ has no symmetric zeros, then $T_{0}$ and $T_{1}$ are both nondegenerate. If $\mathbf{p}$ possesses a symmetric zero a, then these operators are both degenerate, and moreover, $T_{0}^{*}[\bar{a}]=T_{1}^{*}[\bar{a}]=0$.

The proof is based on the following special property of Toeplitz operators.

Lemma 1. Let $\mathbf{p}$ be a polynomial, and let $T_{0}, T_{1}$ be the corresponding operators. Then for any $z \neq 0$ we have

$$
\begin{aligned}
& T_{0}^{*}\left[z^{2}\right]=\alpha \cdot[z]+\beta \cdot[-z], \\
& T_{1}^{*}\left[z^{2}\right]=\frac{1}{z}(\alpha \cdot[z]-\beta \cdot[-z]),
\end{aligned}
$$

where the coefficients are $\alpha=\frac{1}{2} \overline{\mathbf{p}(\bar{z})}$ and $\beta=\frac{1}{2} \overline{\mathbf{p}(-\bar{z})}$.

Proof. See the Appendix. 
Proof of Proposition 1. Suppose that $a$ is a symmetric zero of the polynomial $\mathbf{p}$; substituting $z=\sqrt{\bar{a}}$ (either of the two complex roots of $\bar{a}$ ) in (5), we get $\alpha=\beta=0$, whence $T_{0}^{*}[\bar{a}]=T_{1}^{*}[\bar{a}]=0$.

Now, assume that $\mathbf{p}$ has no symmetric zeros. We show that in this case the operator $T_{0}^{*}$ is nondegenerate; the proof for $T_{1}^{*}$ is similar. First, we treat the case where $\mathbf{p}$ has no multiple roots. Let $z_{1}, \ldots, z_{N}$ be its roots. Applying (5), we get $T_{0}^{*}\left[\bar{z}_{j}^{2}\right]=\frac{1}{2} \overline{\mathbf{p}\left(-z_{j}\right)}\left[-\bar{z}_{j}\right]$ for any $j=1, \ldots, N$. Moreover, we have $\mathbf{p}\left(-z_{j}\right) \neq 0$; otherwise a pair of symmetric zeros $\left\{ \pm z_{j}\right\}$ appears. Observe that the vectors $\left[-\bar{z}_{1}\right], \ldots,\left[-\bar{z}_{N}\right]$ are independent because the numbers $-\bar{z}_{1}, \ldots,-\bar{z}_{N}$ are distinct, so that they form a basis of the space $\mathbb{R}^{N}$. Consequently, the range of the operator $T_{0}^{*}$ coincides with $\mathbb{R}^{N}$, and so this operator is nondegenerate. Now we turn to the general case: the polynomial has roots $z_{1}, \ldots, z_{q}$ of multiplicities $r_{1}, \ldots, r_{q}$. With each root $z_{j}$, we associate the subspace $V_{j}=\operatorname{span}\left\{\left[\bar{z}_{j}^{2}\right]^{l}\right.$, $\left.l=0, \ldots, r_{j}-1\right\} \subset \mathbb{R}^{N}$. Differentiating (5) $r_{j}-1$ times in $z$ and substituting $z=\bar{z}_{j}$, we obtain $T_{0}^{*} V_{j}=\operatorname{span}\left\{\left[-\bar{z}_{j}\right]^{l}, l=0, \ldots, r_{j}-1\right\}$. These $q$ subspaces, one for each of the roots $z_{j}, j=1, \ldots, q$, generate the entire $\mathbb{R}^{N}$. Consequently, $T_{0}^{*}$ is nondegenerate.

We pass to common invariant subspaces of the operators $T_{0}, T_{1}$. For every cyclic set $\mathbf{b}=\left\{b_{1}, \ldots, b_{n}\right\}$, we denote

$$
U_{\mathbf{b}}=\operatorname{span}\left\{\left[\bar{b}_{k}\right], k=1, \ldots, n\right\} .
$$

If $n<N$, then the space $U_{\mathbf{b}}$ is of dimension $n$ and, hence, is a nontrivial subspace in $\mathbb{R}^{N} ;$ for $n \geq N$ it coincides with $\mathbb{R}^{N}$.

If the polynomial $\mathbf{p}$ has a symmetric zero $a$, then the kernels of $T_{0}^{*}, T_{1}^{*}$ possess a common nonzero element $[\bar{a}]$. Consequently, the operators $T_{0}, T_{1}$ have a common invariant hyperplane $[\bar{a}]^{\perp}$, and moreover, $\operatorname{Im} T_{i} \subset[\bar{a}]^{\perp}, i=0,1$. Now, assume that there are no symmetric zeros.

Proposition 2. Suppose $\mathbf{p}$ has no symmetric zeros. Then

a) if $\mathbf{p}$ possesses a cycle $\mathbf{b}=\left\{b_{1}, \ldots, b_{n}\right\}$, then $T_{0}^{*}$ and $T_{1}^{*}$ have a common invariant subspace $U_{\mathbf{b}}$, and both operators are cyclic on this subspace:

$$
T_{0}^{*}\left[\bar{b}_{k+1}\right]=\alpha_{k}\left[\bar{b}_{k}\right], \quad T_{1}^{*}\left[\bar{b}_{k+1}\right]=\beta_{k}\left[\bar{b}_{k}\right], \quad k=1, \ldots, n,
$$

where $\alpha_{k}=\frac{1}{2} \overline{\mathbf{p}\left(b_{k}\right)}, \beta_{k}=\frac{1}{2} \frac{1}{b_{k}} \overline{\mathbf{p}\left(b_{k}\right)}$;

b) any common invariant subspace of the operators $T_{0}^{*}, T_{1}^{*}$ contains the subspace (6) for some cycle $\mathbf{b}$ of the polynomial $\mathbf{p}$; in particular, if $\mathbf{p}$ has no cycles at all, then the pair $T_{0}, T_{1}$ is irreducible.

Thus, if there are no symmetric zeros, but the pair of operators $T_{0}, T_{1}$ turns out to be reducible, then the polynomial must have a cycle $\mathbf{b}$. In this case $U_{\mathbf{b}}^{\perp}$ is a common invariant subspace. The proof of Proposition 2 is based on two lemmas.

Lemma 2. If a polynomial $\mathbf{p}$ has no symmetric zeros, then each eigenvector of the operator $T_{0}^{*}\left(T_{1}^{*}\right)^{-1}$ coincides, up to normalization, with the vector $[z]$ for a suitable $z \in$ $\mathbb{C} \backslash\{0\}$.

Proof. By Proposition 1, the operators $T_{0}^{*}$ and $T_{1}^{*}$ are nondegenerate, whence the operator $T_{0}^{*}\left(T_{1}^{*}\right)^{-1}$ is well defined on $\mathbb{R}^{N}$ and is also nondegenerate. For arbitrary $v \in \mathbb{R}^{N}$, the $i$ th component of the vector $T_{0}^{*} v$ is equal to the $(i+1)$ st component of the vector $T_{1}^{*} v, i=1, \ldots, N-1$. Therefore, for any $y=\left(y_{1}, \ldots, y_{N}\right)^{T} \in \mathbb{R}^{N}$, we have

$$
T_{0}^{*}\left(T_{1}^{*}\right)^{-1} y=\left(y_{2}, \ldots, y_{N}, f(y)\right)^{T},
$$

where $f(y)=\sum_{k} f_{k} y_{k}$ is a linear functional on $\mathbb{R}^{N}$. To prove this, it suffices to take the vector $v=\left(T_{1}^{*}\right)^{-1} y$ and to compare the corresponding coordinates of $T_{0}^{*} v$ and $T_{1}^{*} v$. Each 
eigenvector of the operator (8) has the form $[z]$, where $z$ is the corresponding eigenvalue, which is a root of the characteristic polynomial $P(z)=z^{N}-\sum_{k=1}^{N} f_{k} z^{k-1}$.

Lemma 3. Let $A$ and $B$ be two finite sets of nonzero complex numbers, and let $B$ be nonempty. Suppose that for each $z \in B$ the numbers $\pm \sqrt{z}$ are both in $A \cup B$; then either $A$ contains a pair of symmetric numbers $\{a,-a\}$ or $B$ contains a cyclic set $\mathbf{b}$ such that $-\mathbf{b} \subset A$.

Proof. The proof is outlined in the Appendix.

Proof of Proposition 2, a) If the polynomial $\mathbf{p}(z)$ has a cycle $\mathbf{b}=\left\{b_{1}, \ldots, b_{n}\right\}$, then we substitute $z=\bar{b}_{k}$ in (5) and arrive at (7).

b) If the operators $T_{0}, T_{1}$ possess a common nontrivial invariant subspace, then its orthogonal complement, to be denoted by $U$, is a common invariant subspace of the operators $T_{0}^{*}, T_{1}^{*}$. Since the operators $T_{i}^{*}$ are nondegenerate, it follows that the operator $T_{0}^{*}\left(T_{1}^{*}\right)^{-1}$ has the same invariant subspace $U$; therefore, it has at least one eigenvector in that subspace. By Lemma 2, this eigenvector is of the form $\left[z_{0}\right]$ for some $z_{0} \in \mathbb{C} \backslash\{0\}$. Consider the sets

$$
A=\{z \in \mathbb{C} \backslash\{0\}, \mathbf{p}(\bar{z})=0\}, \quad B=\{z \in \mathbb{C} \backslash\{0\},[z] \in U\}
$$

Clearly, these sets are both finite, and $B$ is nonempty because $z_{0} \in B$. Observe that for any $b \in B$ the two numbers $\pm \sqrt{b}$ are in $A \cup B$. Indeed, if one of the numbers $\pm \sqrt{b}$ (denote that number by $b_{0}$ ) is not in $A$, then we substitute $z=b_{0}$ in (5) and get

$$
\left[b_{0}\right]=\frac{1}{\overline{\mathbf{p}\left(\bar{b}_{0}\right)}}\left(T_{0}^{*}[b]+b_{0} T_{1}^{*}[b]\right),
$$

whence $\left[b_{0}\right] \in U$ and so $b_{0} \in B$. Applying Lemma 3. we conclude that p possesses either a pair of symmetric zeros (which is impossible), or a cycle $\mathbf{b}=\left\{b_{1}, \ldots, b_{n}\right\}$ such that $\left[\bar{b}_{j}\right] \in U, j=1, \ldots, n$.

\section{§3. Cleaning a Polynomial}

Thus, the Toeplitz operators of a clean polynomial are nondegenerate and irreducible. We aim at reducing the general Toeplitz operators to this special case. For this, we devise an algorithm that cleans any polynomial out of its symmetric zeros and cycles.

If $\mathbf{p}$ has either a pair of symmetric zeros $\pm \sqrt{a}$ or a cycle $\mathbf{b}$, then we define $\mathbf{p}_{a}(z)=$ $\frac{z-a}{z^{2}-a} \mathbf{p}(z)$ and $\mathbf{p}_{\mathbf{b}}(z)=\frac{\mathbf{p}(z)}{P_{\mathbf{b}}(z)}$, where $P_{\mathbf{b}}(z)=\prod_{b \in \mathbf{b}}(z+b)$. Let $T_{i}^{(a)}, i=0,1$, be the Toeplitz operators (in the space $\mathbb{R}^{N-1}$ ) associated with the polynomial $\mathbf{p}_{a}$. This means that the $(N-1) \times(N-1)$ matrices of these operators are defined by formulas (1) with the coefficients of the polynomial $\mathbf{p}_{a}$. The operators $T_{i}^{(\mathbf{b})}$ acting in $\mathbb{R}^{N-n}$ and corresponding to the polynomial $\mathbf{p}_{\mathbf{b}}$ are defined similarly.

We say that linear operators $A_{0}, A_{1}$ in a space $L$ are isomorphic to operators $A_{0}^{\prime}, A_{1}^{\prime}$ in a space $L^{\prime}$ if these spaces have the same dimension and there exists a basis of $L$ and a basis of $L^{\prime}$, in which the matrix of $A_{i}$ is equal to that of $A_{i}^{\prime}$ for $i=0,1$.

If a polynomial $\mathbf{p}$ has a symmetric zero $a$, then the operators $T_{0}, T_{1}$ possess a common invariant subspace $[\bar{a}]^{\perp}$ (see Proposition 1). Now we clarify how these operators act on 
that subspace. We define the following system of vectors in $\mathbb{R}^{N}$ :

$$
\begin{aligned}
\tilde{e}_{1} & =(a,-1,0,0, \ldots, 0)^{T}, \\
\tilde{e}_{2} & =(0, a,-1,0, \ldots, 0)^{T}, \\
& \vdots \\
\tilde{e}_{N-1} & =(0, \ldots, 0, a,-1)^{T} .
\end{aligned}
$$

These vectors belong to the subspace $[\bar{a}]^{\perp}$ and form its basis.

Proposition 3. If a polynomial $\mathbf{p}$ has a symmetric zero a, then the restrictions of the operators $T_{0}, T_{1}$ to the subspace $[\bar{a}]^{\perp}$ are isomorphic to the operators $T_{0}^{(a)}, T_{1}^{(a)}$. In the basis (9), the matrices $\left.T_{i}\right|_{[\bar{a}] \perp}$ have the form $T_{i}^{(a)}, i=0,1$.

Proof. Consider the $N \times(N-1)$ matrix $C_{a}$ formed by the column vectors $\tilde{e}_{1}, \ldots, \tilde{e}_{N-1}$ defined in (9). A direct calculation shows that

$$
C_{a} T_{i}^{(a)}=T_{i} C_{a}, \quad i=0,1
$$

(this is equivalent to the identity $\left.\left(z^{2}-a\right) \mathbf{p}_{a}(z)=(z-a) \mathbf{p}(z)\right)$. Hence, the matrices of the operators $T_{i}$ in the new basis coincide with the matrices $T_{i}^{(a)}$.

For a given cyclic set $\mathbf{b}=\left\{b_{1}, \ldots, b_{n}\right\}$, we define

$$
Q_{\mathbf{b}}(z)=\prod_{j=1}^{n}\left(z-z_{j}\right)=\sum_{k=0}^{n} q_{k} z^{k} .
$$

Clearly, $P_{\mathbf{b}}(z)=Q_{\mathbf{b}}\left(z^{2}\right) / Q_{\mathbf{b}}(z)$. Consider the following system of vectors in $\mathbb{R}^{N}$ :

$$
\tilde{e}_{1}=\left(q_{0}, q_{1}, \ldots, q_{n}, 0, \ldots, 0\right)^{T},
$$

$$
\tilde{e}_{N-n}=\left(0, \ldots, 0, q_{0}, q_{1}, \ldots, q_{n}\right)^{T} .
$$

For each $b \in \mathbf{b}$ we have $\left(\tilde{e}_{j},[\bar{b}]\right)=b^{j-1} Q_{\mathbf{b}}(b)=0$. This shows that the vectors $\tilde{e}_{j}$, $j=1, \ldots, N-n$, belong to the subspace $U_{\mathbf{b}}^{\perp}$. Since they are independent, they constitute a basis of this subspace.

Proposition 4. If $\mathbf{b}=\left\{b_{1}, \ldots, b_{n}\right\}$ is a cycle of the polynomial $\mathbf{p}$, then the restrictions of the operators $T_{0}, T_{1}$ to the subspace $U_{\mathbf{b}}^{\perp}$ are isomorphic to the operators $T_{0}^{(\mathbf{b})}, T_{1}^{(\mathbf{b})}$. For the basis (11), the matrices of the operators $\left.T_{i}\right|_{U_{\mathbf{b}}^{\perp}}$ coincide with the matrices $T_{i}^{(\mathbf{b})}$ $(i=0,1)$.

Proof. Let $\left\{p_{k}\right\}_{k=0, \ldots, N}$ and $\left\{p_{k}^{\prime}\right\}_{k=0, \ldots, N-n}$ be the coefficients of the polynomials $\mathbf{p}$ and $\mathbf{p}_{\mathbf{b}}$, respectively. We complement each of these sequences by zeros in both directions to infinity. Since $\mathbf{p}(z)=\mathbf{p}_{\mathbf{b}}(z) P_{\mathbf{b}}(z)$, it follows that $\mathbf{p}(z) Q_{\mathbf{b}}(z)=\mathbf{p}_{\mathbf{b}}(z) Q_{\mathbf{b}}\left(z^{2}\right)$. Comparing the coefficients corresponding to the term $z^{r}$, we get $\sum_{s \in \mathbb{Z}} p_{r-s} q_{s}=\sum_{t \in \mathbb{Z}} p_{r-2 t}^{\prime} q_{t}$. We take arbitrary $i \in\{1, \ldots, N\}$ and $j=\{1, \ldots, N-n\}$, and set $r=2 i-j-1, k=s+j$, $l=i-t$, obtaining

$$
\sum_{k} p_{2 i-k-1} q_{k-j}=\sum_{l} p_{2 l-j-1}^{\prime} q_{i-l} .
$$

This identity means that the elements of the matrices $T_{0} C_{\mathbf{b}}$ and $C_{\mathbf{b}} T_{0}^{(\mathbf{b})}$ in the $i$ th row and $j$ th column are equal, where $C_{\mathbf{b}}$ is the $N \times(N-n)$ matrix formed by the column 
vectors $\tilde{e}_{1}, \ldots, \tilde{e}_{N-n}$ defined in (11). Thus, $T_{0} C_{\mathbf{b}}=C_{\mathbf{b}} T_{0}^{(\mathbf{b})}$, as required. The proof for the operators $T_{1}, T_{1}^{(\mathbf{b})}$ is the same, with the replacement of $r=2 i-j$ by $r=2 i-j-1$.

Applying Propositions 3 and 4 to an arbitrary polynomial p, we can consecutively remove all its symmetric zeros and all its cycles, obtaining a clean polynomial $\tilde{\mathbf{p}}$. This is the process of cleaning. If $\mathbf{p}$ possesses a symmetric zero $a$, then we pass to the polynomial $\mathbf{p}_{a}(z)=\frac{z-a}{z^{2}-a} \mathbf{p}(z)$. This reduces the degree by one. If $\mathbf{p}_{a}$ has a symmetric zero (perhaps, again $a$ ), then we do the same, and so on, until we obtain some polynomial $\mathbf{p}_{0}(z)$ without symmetric zeros. Suppose we have removed the symmetric zeros $a_{1}, \ldots, a_{t}$ (counted with multiplicities). Then we start removing cycles. Suppose $\mathbf{p}_{0}$ has cycles $\mathbf{b}_{0}, \ldots, \mathbf{b}_{s}$ of multiplicities $r_{0}, \ldots, r_{s}$, respectively. Throughout the paper, $\mathbf{b}_{0}$ denotes the trivial cycle; the others are nontrivial and distinct. For simplicity, we use the notation $P_{\mathbf{b}_{j}}(z)=P_{j}(z)$. The polynomial

$$
\tilde{\mathbf{p}}(z)=\mathbf{p}_{0}(z) / \prod_{j=0}^{s} P_{j}^{r_{j}}(z)
$$

has no cycles. It has no symmetric zeros either, because its roots are among the roots of $\mathbf{p}_{0}$. This means that $\tilde{\mathbf{p}}(z)$ is a clean polynomial.

We note that the result is independent of the order of removing the cycles. Indeed, since different cycles are disjoint, removing one of them does not affect the others. However, the uniqueness of the first passage (from the initial polynomial $\mathbf{p}$ to $\mathbf{p}_{0}$ ) is not that obvious. At each step the polynomial obtains a new root: a pair of roots $\pm \sqrt{a}$ is replaced with the root $a$. Hence, new pairs of symmetric zeros and new cycles may arise. For example, the polynomial $\mathbf{p}(z)=\left(z^{2}-4\right)(z+4)$ has only one pair of symmetric zeros, $z= \pm 2$. However, removing this pair we pass to the polynomial $\mathbf{p}_{a}(z)=(z-4)(z+4)$, which has another pair: $z= \pm 4$. Moreover, the polynomial may have several pairs of symmetric zeros, and at each step we have the choice which one to remove for the next passage. Nonetheless, the polynomial $\tilde{\mathbf{p}}$ is determined in a unique way by the initial polynomial p. To show this, we need one more notion. Given $a \in \mathbb{C} \backslash\{0\}$, we denote by $\mathcal{T}_{a}$ the binary tree with the root $a^{2}$. The root is located at the zero level and has valency 1 ; all other vertices have valency 2 . The first level contains one vertex with number $a$, the second contains two vertices $\pm \sqrt{a}$, and so on. Every vertex $z$ has two neighbors $\pm \sqrt{z}$ at the next level. Let $\mathcal{A}$ be a finite subset of vertices different from the root. An element of $\mathcal{A}$ may be repeated, in which case it is counted with its multiplicity. The set $\mathcal{A}$ is called a cut set of multiplicity $l \geq 1$ if any infinite path starting at the root has precisely $l$ common vertices with $\mathcal{A}$, counted with their multiplicities. Any cut set is finite. If the multiplicity of a cut set is not mentioned, it is 1 . The simplest cut set $\mathcal{A}=\{a\}$ will be called trivial.

Let $Z=\left\{z_{1}, \ldots, z_{N}\right\}$ be a set of nonzero complex numbers. Some numbers may coincide, and then they are counted with multiplicity. Two sets coincide if their elements with the corresponding multiplicities coincide. We call a set $Z_{0}=\left\{h_{1}, \ldots, h_{n}\right\}$ a root set for $Z$ if

a) $Z_{0}$ has no pairs of symmetric numbers (in other words, the sum of any two elements of $Z_{0}$ is not zero);

b) the set $Z$ can be split into subsets $\mathcal{A}_{1}, \ldots, \mathcal{A}_{n}$ (respecting the multiplicities of elements) so that $\mathcal{A}_{k}$ is a cut set of the tree $\mathcal{T}_{h_{k}}, k=1, \ldots, n$.

If all the cut sets $\mathcal{A}_{i}$ are trivial, then $Z_{0}=Z$. If, for instance, $\mathcal{A}_{1}$ is nontrivial, then $Z$ contains a pair of symmetric numbers $\pm z$. After passage to the preceding level (replacement of the pair $\{z,-z\}$ by one element $z^{2}$ ), we obtain a cut set $\mathcal{A}_{1}^{\prime}$ of the same tree. The total number of elements of $Z$ decreases by 1 . Finitely many iterations of the 


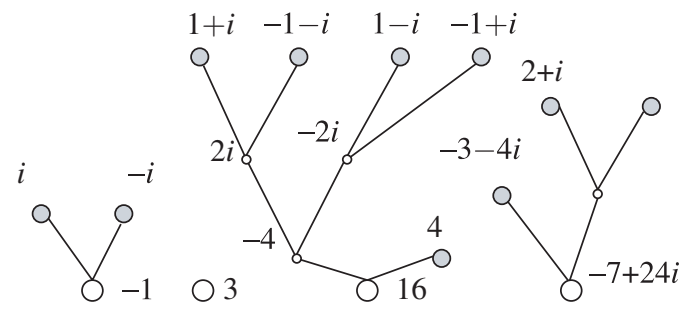

Figure 1. Example of a set $Z$ with the root set $Z_{0}=\{-1,3,16,-7+24 i\}$.

algorithm take the entire set $\mathcal{A}_{1}$ to the root $z_{1}$ of the corresponding tree. The same can be done with the other cut sets. Thus, any set $Z$ can be transformed to its root set $Z_{0}$ by finitely many steps (removals of symmetric numbers).

Lemma 4. For any $Z$ there exists a unique root set.

Proof. See the Appendix.

In Figure 1, the set $Z$ consists of 11 elements: $\{ \pm i, 1 \pm i,-1 \pm i, \pm(2+i), 3,4,-3-4 i\}$; its root set is $Z_{0}=\{-1,3,16,-7+24 i\}$.

Thus, the elements of $Z_{0}$ and their multiplicities are uniquely determined by the set $Z$. We note that, in general, the cut sets $\mathcal{A}_{k}$ can be chosen in many ways, and the partition $Z=\bigcup_{k} \mathcal{A}_{k}$ is not unique.

Example 1. For the set $Z=\left\{e^{\frac{\pi i}{3}}, e^{\frac{2 \pi i}{3}}, e^{\frac{4 \pi i}{3}}, e^{\frac{5 \pi i}{3}}\right\}$, the root set is $Z_{0}=\left\{e^{\frac{2 \pi i}{3}}, e^{\frac{4 \pi i}{3}}\right\}$. Taking the pair of symmetric zeros $\left\{e^{\frac{\pi i}{3}}, e^{\frac{4 \pi i}{3}}\right\}$ at the first step, we get consecutively

$$
Z \rightarrow Z^{\prime}=\left\{e^{\frac{2 \pi i}{3}}, e^{\frac{2 \pi i}{3}}, e^{\frac{5 \pi i}{3}}\right\} \rightarrow Z_{0} .
$$

If, however, we take the pair $\left\{e^{\frac{2 \pi i}{3}}, e^{\frac{5 \pi i}{3}}\right\}$ at the first step, then

$$
Z \rightarrow Z^{\prime \prime}=\left\{e^{\frac{\pi i}{3}}, e^{\frac{4 \pi i}{3}}, e^{\frac{4 \pi i}{3}}\right\} \rightarrow Z_{0} .
$$

Note that the intermediate sets $Z^{\prime}$ and $Z^{\prime \prime}$ have no common elements at all.

Let $Z=\left\{z_{1}, \ldots, z_{N}\right\}$ be the set of roots of the polynomial $\mathbf{p}$, counted with their multiplicity. The polynomial $\mathbf{p}_{0}$ is well defined and is given by the formula

$$
\mathbf{p}_{0}(z)=p_{N} \prod_{z_{j} \in Z_{0}}\left(z-z_{j}\right)
$$

where $Z_{0}$ is the root set of $Z$. If we remove all the cycles $\mathbf{b}_{1}, \ldots, \mathbf{b}_{s}, \mathbf{b}_{0}$ of the polynomial $\mathbf{p}_{0}$, we arrive at the clean polynomial (12). The trivial cycle $\mathbf{b}_{0}$ will always be deleted last. By Propositions 3 and 4 , the matrices of the operators $T_{0}, T_{1}$ are transformed consecutively with the help of the following transfer matrices:

$$
C_{a_{1}}, \ldots, C_{a_{t}}, C_{\mathbf{b}_{1}, 1}, \ldots, C_{\mathbf{b}_{1}, r_{1}}, \ldots, C_{\mathbf{b}_{s}, 1}, \ldots, C_{\mathbf{b}_{s}, r_{s}}, C_{\mathbf{b}_{0}, 1}, \ldots, C_{\mathbf{b}_{0}, r_{0}} .
$$

The matrices $C_{a_{k}}$ were defined in the proof of Proposition 3. The matrix $C_{a_{k}}$ corresponding to a symmetric zero $a_{k}$ is formed by the column vectors (9). The dimension $N$ depends on the matrix $C_{a_{k}}$ and is equal to the degree of the polynomial $\mathbf{p}$ at the current step, i.e., by the moment of removing the symmetric zero $a_{k}$. The situation with the matrices $C_{\mathbf{b}_{j}, k}$ is quite similar. For a given cycle $\mathbf{b}_{j}$, the matrix $C_{\mathbf{b}_{j}, k}$ is formed by the column vectors (11) corresponding to $\mathbf{b}_{j}$. The dimensions $N$ are different for all the matrices $C_{\mathbf{b}_{j}, k}, k=1, \ldots, r_{j}$. For each $k$, this dimension is equal to the degree of the polynomial $\mathbf{p}$ at the current step, i.e., by the moment of the $k$ th removal of the cycle $\mathbf{b}_{j}$ (since its multiplicity is $r_{j}$, it must be removed $r_{j}$ times). 
As a result, we obtain the restrictions of the operators $T_{0}, T_{1}$ to their common invariant subspace $\tilde{V}$ spanned by the columns of the matrix

$$
\tilde{C}=C_{\mathbf{b}_{0}, r_{0}} \cdots C_{\mathbf{b}_{0}, 1} \cdot C_{\mathbf{b}_{s}, r_{s}} \cdots C_{\mathbf{b}_{s}, 1} \cdot C_{\mathbf{b}_{1}, r_{1}} \cdots C_{\mathbf{b}_{1}, 1} \cdot C_{a_{t}} \cdots C_{a_{1}} .
$$

In the basis formed by the columns of the matrix $\tilde{C}$, the operators $\left.T_{i}\right|_{V_{0}}, i=0,1$, are isomorphic to the operators $\tilde{T}_{i}, i=0,1$, corresponding to the clean polynomial $\tilde{\mathbf{p}}$. We summarize the results obtained as follows.

Theorem 2. For an arbitrary polynomial $\mathbf{p}(z)=c_{N} \prod_{z_{k} \in \mathbb{Z}}\left(z-z_{k}\right)$, the corresponding clean polynomial $\tilde{\mathbf{p}}$ is given by formula (12), where $\mathbf{p}_{0}$ is defined by (13). Both the clean polynomial and the matrix (14) are independent of the order in which the symmetric zeros and the cycles have been removed. Furthermore, we have $T_{i} \tilde{C}=\tilde{C} \tilde{T}_{i}, i=0,1$, where the $\tilde{T}_{i}, i=0,1$, are the Toeplitz operators corresponding to the polynomial $\tilde{\mathbf{p}}$.

Thus, the space $\tilde{V}$ spanned by the columns of the matrix $\tilde{C}$ is invariant with respect to the operators $T_{i}, i=0,1$. In the basis formed by the columns of $\tilde{C}$, the matrices of the operators $T_{i}$ coincide with the matrices $\tilde{T}_{i}, i=0,1$.

\section{§4. Kernels And InVAriant SubspaCES OF $T_{0}^{*}, T_{1}^{*}$}

For a linear operator $B$ and a natural number $n$, we denote by $E_{n}$ the $n$th root subspace of $B: E_{n}=\left\{u \in \mathbb{R}^{N}, B^{n} u=0\right\}$. In particular, $E_{1}=\operatorname{Ker} B$. It turns out that for any polynomial $\mathbf{p}$ the kernels of the operators $T_{0}^{*}$ and $T_{1}^{*}$ coincide, and all their root subspaces also coincide.

Theorem 3. For any polynomial $\mathbf{p}$, the two operators $T_{0}^{*}$ and $T_{1}^{*}$ have one and the same kernel; it is spanned by the vectors $[\bar{a}]^{l}$, where a is a symmetric zero of $\mathbf{p}$ of multiplicity $r_{a}$, and $l=0, \ldots, r_{a}-1$.

Moreover, for every $n \geq 1$ the two operators $T_{0}^{*}$ and $T_{1}^{*}$ have one and the same root subspace $E_{n}$. It is spanned by the vectors $[\bar{\lambda}]^{l}$, where the complex numbers $\lambda$ are such that, at the levels from the first to the $n$th inclusive, each tree $\mathcal{T}_{\lambda}$ has a cut set consisting of roots of $\mathbf{p}$ (counted with multiplicity), and $r_{\lambda} \geq 1$ is the multiplicity of that cut set, $l=0, \ldots, r_{\lambda}-1$.

Proof. We use induction on $N$. We show that the kernel and the root subspaces of the matrix $T_{0}^{*}$ have the form indicated. The proof for $T_{1}^{*}$ is the same.

For $N=1$ the matrices $T_{i}^{*}$ are both diagonal with the entries $p_{0}, p_{1}$; therefore, these matrices are nonsingular, because $p_{0} p_{1} \neq 0$. Assume that the claim is true for the matrices of dimension at most $N-1$. Consider the operators $T_{0}^{*}, T_{1}^{*}$ of dimension $N$. If they are both nonsingular, there is nothing to prove. If at least one of them has a nontrivial kernel, then, by Proposition 1, the polynomial $\mathbf{p}$ has a symmetric zero $a$. Furthermore, the vector $[\bar{a}]$ belongs to the intersection of the kernels, and the restrictions of the operators $T_{i}$ to the subspace $[\bar{a}]^{\perp}$ are isomorphic to the operators $T_{i}^{(a)}$.

Now we apply the inductive hypothesis to the operators $T_{i}^{(a)}$. Their adjoint operators (in the basis $\tilde{e}_{1}, \ldots, \tilde{e}_{N-1}$ defined by (9)) have common invariant subspaces $E_{n}^{\prime}, n \geq 0$. They are related to the root subspaces $E_{n}^{\prime}$ of the operators $T_{i}$ by the formula $E_{n}^{\prime}=C_{a}^{*} E_{n}^{\prime}$, where the transfer matrix $C_{a}$ is formed by the columns $\left\{\tilde{e}_{j}\right\}$. The subspace $E_{n}^{\prime}$ is spanned by the vectors $[\bar{\lambda}]^{l}$, where the numbers $\lambda$ are such that, at the levels from the first to the $n$th inclusive, the binary tree $\mathcal{T}_{\lambda}$ has a cut set that consists of roots of the polynomial $\mathbf{p}_{a}, l=0, \ldots, r_{\lambda}-1$, where $r_{\lambda}$ is the multiplicity of that cut set. Take any of these numbers $\lambda$. Suppose that $a \notin \mathcal{T}_{\lambda}$; then the polynomial $\mathbf{p}$ has the same roots on the tree as the polynomial $\mathbf{p}_{a}$. Furthermore, the space $\operatorname{span}\left\{[\bar{\lambda}]^{l} \in \mathbb{R}^{N}, l \leq r_{\lambda}-1\right\}$ is mapped 
isomorphically by the operator $C_{a}^{*}$ to a similar space in $\mathbb{R}^{N-1}$. This proves the theorem for the values of $\lambda$ for which the tree does not contain $a$.

Now, assume that $a$ belongs to the tree $\mathcal{T}_{\lambda}$. Then the passage from $\mathbf{p}$ to $\mathbf{p}_{a}$ takes the pair of symmetric zeros $\pm \sqrt{a}$, which are the vertices of $\mathcal{T}_{\lambda}$ neighboring with $a$, to one vertex $a$. Consequently, all cut sets that consist of roots of $\mathbf{p}$ become cut sets consisting of roots of $\mathbf{p}_{a}$. If $a \neq \lambda$, then the space $\operatorname{span}\left\{[\bar{\lambda}]^{l} \in \mathbb{R}^{N}, l \leq r_{\lambda}-1\right\}$ is mapped isomorphically to a similar space in $\mathbb{R}^{N-1}$ by the operator $C_{a}^{*}$. Therefore, in this case the theorem follows.

It remains to consider the case where $a=\lambda$. Then the subspace in question loses one dimension and becomes the space $\operatorname{span}\left\{[\bar{\lambda}]^{l} \in \mathbb{R}^{N-1}, l \leq r_{\lambda}-2\right\}$ (or disappears at all if $r_{\lambda}=1$ ). However, in this case the multiplicity $r_{\lambda}$ also reduces by 1 , because one of the cut sets, namely $\{ \pm \sqrt{\lambda}\}$, comes to the zero level (to the root) and is not counted anymore. This completes the proof.

Thus, the adjoint operators $T_{0}^{*}$ and $T_{1}^{*}$ have common root subspaces. Let $E_{q}$ be the largest of these subspaces. This means that $E_{q+1}=E_{q}$. It follows that for any indices $d_{1}, \ldots, d_{q} \in\{0,1\}$ we have $T_{d_{1}}^{*} \cdots T_{d_{q}}^{*} E_{q}=0$. Consequently,

$$
\operatorname{Im}\left(T_{d_{1}} \cdots T_{d_{s}} E_{q}^{\perp}\right)=E_{q}^{\perp}
$$

for any $s \geq q$ and any indices $d_{1}, \ldots, d_{s} \in\{0,1\}$. Thus, $E_{q}^{\perp}$ is a common invariant subspace of the operators $T_{0}, T_{1}$, and both of them are nondegenerate on this subspace.

Proposition 5. The largest root subspace $E_{q}$ of the operators $T_{0}^{*}, T_{1}^{*}$ is spanned by the vectors $[\bar{\lambda}]^{l}$, where the complex numbers $\lambda$ are such that the binary tree $\mathcal{T}_{\lambda}$ possesses a cut set of multiplicity $r_{\lambda} \geq 1$ that consists of roots of the polynomial $\mathbf{p}, l=1, \ldots, r_{\lambda}-1$.

On the subspace $E_{q}^{\perp}$ the operators $T_{0}, T_{1}$ are both nondegenerate and are isomorphic to the operators $T_{0}^{(0)}, T_{1}^{(0)}$ corresponding to the polynomial $\mathbf{p}_{0}$. The transfer matrix is $C_{0}=C_{a_{t}} \cdots C_{a_{1}}$, where $a_{1}, \ldots, a_{t}$ are the symmetric zeros removed consecutively by the cleaning algorithm.

\section{$\S 5$. Common invariant subspaces of $T_{0}, T_{1}$}

Now we are able to make a complete list of common invariant subspaces of the Toeplitz operators $T_{0}, T_{1}$. This will be done in terms of generalized cycles of the polynomial $\mathbf{p}$.

For a given cyclic set $\mathbf{b}$, the cyclic tree $\mathcal{T}_{\mathbf{b}}$ is constructed as follows. The elements of $\mathbf{b}$ form the root of the cyclic tree. They are elements of the zero level. At every vertex $z$ of the root $\mathbf{b}$ one edge starts, leading to a neighboring vertex of the first level. One of the numbers $\pm \sqrt{z}$ does not belong to $\mathbf{b}$; this number is associated with the neighboring vertex of the first level. At every vertex $z$ that does not belong to the root, two edges start; they lead to the vertices $\pm \sqrt{z}$ of the next level. The tree has $n$ vertices of the zero level and $2^{l-1} n$ vertices of the $l$ th level for each $l \geq 1$. The root $\mathbf{b}=\{1\}$ and the corresponding tree are said to be trivial; all others are nontrivial. The tree with the trivial root $\mathcal{T}_{\{1\}}$ is the usual binary tree $\mathcal{T}_{-1}$ considered above.

A set $\mathcal{A}$ of vertices of the tree is called a cut set of multiplicity $r \geq 1$ if

a) $\mathcal{A}$ contains no elements of the root;

b) any infinite path from each vertex of the root (all the paths are "upward", i.e., with increasing levels) contains exactly $r$ elements of $\mathcal{A}$ (counting with multiplicity).

A generalized cycle of multiplicity $r$ of a polynomial $\mathbf{p}(z)$ is a cyclic set $\mathbf{b}$ such that the corresponding cyclic tree $\mathcal{T}_{\mathbf{b}}$ has a cut set of multiplicity $r$ consisting of roots of the polynomial. The generalized cycle $\mathbf{b}=\{1\}$ is trivial; all other are nontrivial. 


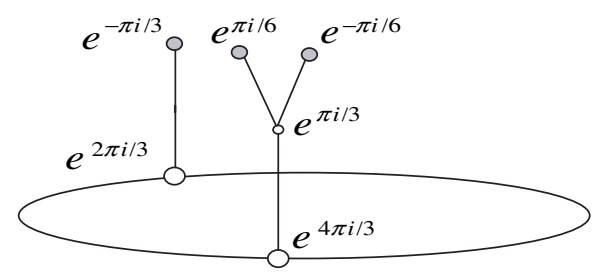

Figure 2. The generalized cycle $\mathbf{b}=\left\{e^{\frac{2 \pi i}{3}}, e^{\frac{4 \pi i}{3}}\right\}$ with the cut set $\mathcal{A}=$ $\left\{e^{-\frac{\pi i}{3}}, e^{\frac{\pi i}{6}},-e^{\frac{\pi i}{6}}\right\}$.

Example 2. The polynomial $\mathbf{p}(z)=\left(z-e^{-\frac{\pi i}{3}}\right)\left(z^{2}-e^{\frac{\pi i}{3}}\right)$ has no regular cycles; however, it has a generalized cycle $\mathbf{b}=\left\{e^{\frac{2 \pi i}{3}}, e^{\frac{4 \pi i}{3}}\right\}$. Indeed, it has three roots on the unit circle: $e^{-\frac{\pi i}{3}}, e^{\frac{\pi i}{6}},-e^{\frac{\pi i}{6}}$. They form a cut set $\mathcal{A}$ of the cyclic tree $\mathcal{T}_{\mathbf{b}}$ (see Figure 2).

Let $\mathbf{b}_{0}, \ldots, \mathbf{b}_{s}$ be generalized cycles of the polynomial $\mathbf{p}$, and let $r_{0}, \ldots, r_{s}$ be their multiplicities. We say that a complex number $\lambda$ is blocked with multiplicity $r$ if the tree $\mathcal{T}_{\lambda}$ has a cut set of multiplicity $r$ consisting of roots of the polynomial p. A common invariant subspace is said to be indecomposable if it is not a direct sum of common invariant subspaces of smaller dimensions.

Theorem 4. Every indecomposable common invariant subspace of the operators $T_{0}^{*}, T_{1}^{*}$ belongs to one of the following families:

1) $\operatorname{span}\left\{[\bar{\lambda}]^{l}, l=0, \ldots, k-1, \lambda \in \mathcal{B}_{\lambda}\right\}$, where $\lambda$ is an arbitrary element blocked with some multiplicity $r_{\lambda} \geq 1, k=1, \ldots, r_{\lambda}$, and $\mathcal{B}_{\lambda}$ is the smallest (by inclusion) subset of the tree $\mathcal{T}_{\lambda}$ containing $\lambda$ and blocked with multiplicity $k$;

2) $\operatorname{span}\left\{[\bar{\lambda}]^{l}, l=0, \ldots, k-1, \lambda \in \mathcal{B}_{j, k}\right\}$, where $k=1, \ldots, r_{j}, \mathcal{B}_{j, k}$ is the smallest (by inclusion) subset of the cyclic tree $\mathcal{T}_{\mathbf{b}_{j}}$ that contains $\mathbf{b}_{j}$ and is blocked with multiplicity $k$, and $j=0, \ldots, s$.

All the subspaces of the first family lie in the root subspaces of the operators $T_{0}^{*}, T_{1}^{*}$. On each of them the pair $T_{0}^{*}, T_{1}^{*}$ is nilpotent: any operator product of length $N$ vanishes. This family is empty if $\mathbf{p}(z)$ has no symmetric zeros. The second family corresponds to the generalized cycles of the polynomial $\mathbf{p}(z)$. All common invariant subspaces of the operators $T_{0}^{*}, T_{1}^{*}$ are direct sums of several spaces of these two families. The common invariant subspaces of $T_{0}, T_{1}$ are their orthogonal complements.

Corollary 1. If a polynomial $\mathbf{p}$ has no symmetric zeros, then any indecomposable common invariant subspace of the operators $T_{0}^{*}$ and $T_{1}^{*}$ belongs to the family

$$
U_{\mathbf{b}, k}=\operatorname{span}\left\{[\bar{b}]^{l}, l=0, \ldots, k-1, b \in \mathbf{b}\right\},
$$

where $\mathbf{b}$ is an arbitrary cycle of the polynomial $\mathbf{p}, r_{\mathbf{b}}$ is its multiplicity, and $k=1, \ldots, r_{\mathbf{b}}$.

Proof of Theorem 4. We use induction on the dimension $N$. We show the proof for the operator $T_{0}^{*}$; for $T_{1}^{*}$ the arguments are the same. If $N=1$, then the operators are onedimensional, so the claim is trivial. Assuming it for all dimensions not exceeding $N-1$, we consider the operators $T_{0}^{*}, T_{1}^{*}$ of dimension $N$. If at least one of them is degenerate, then, by Proposition 1 the polynomial $\mathbf{p}$ has a symmetric zero $a$. Having passed to the polynomial $\mathbf{p}_{a}$, we apply the inductive hypothesis to the operators $T_{i}^{(a)}, i=0,1$, acting in $[\bar{a}]^{\perp}$ (see Proposition [3). A direct calculation shows that the reverse passage from $\mathbf{p}_{a}$ to $\mathbf{p}$ maps the two families of common invariant subspaces for the operators $\left[T_{i}^{(a)}\right]^{*}$, $i=0,1$, to the corresponding families for the operators $T_{i}^{*}, i=0,1$. 
If both operators $T_{i}^{*}$ are nondegenerate, then the polynomial $\mathbf{p}(z)$ has no symmetric zeros. If it has no cycles either, then it is clean. In this case, by Theorem 1 , the operators $T_{i}^{*}$ have no common invariant subspaces, and there is nothing to prove. If $\mathbf{p}$ has a cycle $\mathbf{b}_{1}$, then the operators $T_{i}^{*}$ possess a common invariant subspace $U_{\mathbf{b}_{1}}=\operatorname{span}\left\{[\bar{b}], b \in \mathbf{b}_{1}\right\}$. Moreover, Propositions 2 and 4 imply that the operators $T_{i}$ restricted to the orthogonal complement $U_{\mathbf{b}_{1}}^{\perp}$ are isomorphic to the operators $T_{i}^{\left(\mathbf{b}_{1}\right)}$. The transfer matrix is $C_{\mathbf{b}_{1}}$. By the inductive hypothesis, all indecomposable common invariant subspaces of the operators $\left[T_{i}^{\left(\mathbf{b}_{1}\right)}\right]^{*}$ belong to family (16) for the polynomial $\mathbf{p}_{\mathbf{b}}$. Consider an arbitrary subspace $U_{\mathbf{b}, k}$ in this family. This is a subspace of $\mathbb{R}^{N-1}$, and we denote it by $U_{\mathbf{b}, k, N-1}$; the corresponding subspace of $\mathbb{R}^{N}$ will be denoted by $U_{\mathbf{b}, k, N}$. Since

$$
U_{\mathbf{b}, k, N-1}= \begin{cases}C_{\mathbf{b}_{1}}^{*} U_{\mathbf{b}, k, N} & \text { if } \mathbf{b} \neq \mathbf{b}_{1}, \\ C_{\mathbf{b}_{1}}^{*} U_{\mathbf{b}, k+1, N} & \text { if } \mathbf{b}=\mathbf{b}_{1},\end{cases}
$$

it follows that the operators $T_{i}^{*}, i=0,1$, have the following system of common invariant spaces:

$\left\{U_{\mathbf{b}, k, N}, \mathbf{b}\right.$ is a cycle of the polynomial $\left.\mathbf{p}, k \leq r_{\mathbf{b}}-1\right\}$.

All these subspaces are indecomposable. Indeed, by Proposition 2 every common invariant subspace of the operators $T_{i}^{*}, i=0,1$, contains the subspace $U_{\mathbf{b}_{2}}$, where $\mathbf{b}_{2}$ is a cycle of $\mathbf{p}(z)$. Therefore, any two common invariant subspaces lying in $U_{\mathbf{b}, r, N}$ contain $U_{\mathbf{b}}$ and, therefore, have a nontrivial intersection. This shows that $U_{\mathbf{b}, k, N}$ is indecomposable. The operators $T_{0}^{*}, T_{1}^{*}$ have no other indecomposable common invariant subspaces. Indeed, any such subspace $L$ contains a subspace $U_{\mathbf{b}_{0}}$ generated by some cycle $\mathbf{b}_{2}$. In case $L=U_{\mathbf{b}_{2}}$, the theorem follows. If $L \neq U_{\mathbf{b}_{2}}$, then we consider the restrictions of the operators $T_{i}^{*}$ to $U_{\mathbf{b}}$ and conclude that $C_{\mathbf{b}}^{*} L$ is an indecomposable common invariant subspace of the operators $\left[T_{i}^{(\mathbf{b})}\right]^{*}, i=0,1$. By the inductive hypothesis, we have $C_{\mathbf{b}}^{*} L=U_{\mathbf{b}, k, N-1}$ for some $\mathbf{b}$ and $n$. Using (17), we deduce that $\mathbf{b}=\mathbf{b}_{2}$ and $L=U_{\mathbf{b}_{2}, k+1, N}$ (if $\mathbf{b} \neq \mathbf{b}_{2}$, then the same identity implies $L=U_{\mathbf{b}, k, N}$, but this subspace does not contain $U_{\mathbf{b}_{2}}$ ).

\section{$\S 6$. The structure of the operators $T_{0}, T_{1}$}

In this section we obtain a complete spectral resolution of a pair of Toeplitz operators in the system of their common invariant subspaces. We start with the case where the polynomial $\mathbf{p}$ has a cycle $\mathbf{b}=\left\{b_{1}, \ldots, b_{n}\right\}$. Proposition 2 says that the operators $T_{0}^{*}, T_{1}^{*}$ are cyclic on the subspace $U_{\mathbf{b}}=\operatorname{span}\left\{\left[\bar{b}_{j}\right], b_{j} \in \mathbf{b}\right\}$ and act by formula (7): the basic vectors $\left[\bar{b}_{j}\right], j=1, \ldots, n$, are mapped to each other cyclically, with coefficients $\alpha_{j}$ (for the operator $T_{0}^{*}$ ) and $\beta_{j}$ (for $T_{1}^{*}$ ). For any polynomial $\mathbf{p}(z)$ and any cyclic set $\mathbf{g}$, we define $\gamma_{\mathbf{g}}(\mathbf{p})=\prod_{g \in \mathbf{g}} \overline{\mathbf{p}(g)}$. Then $\left.\left[T_{0}^{*}\right]^{n}\right|_{U_{\mathbf{b}}}=\left.\left[T_{1}^{*}\right]^{n}\right|_{U_{\mathbf{b}}}=2^{-n} \gamma_{\mathbf{b}}(\mathbf{p}) \cdot I_{n}$, where $I_{n}$ is the identity operator in $\mathbb{R}^{n}$. For $T_{0}$ this is a straightforward consequence of (7), and for $T_{1}$ we also use the identity $\prod_{j=1}^{n} b_{j}=1$. This identity is true because $\prod_{b_{j} \in \mathbf{b}} b_{j}=$ $\prod_{b_{j} \in \mathbf{b}} b_{j+1}=\prod_{b_{j} \in \mathbf{b}} b_{j}^{2}=\left(\prod_{b_{j} \in \mathbf{b}} b_{j}\right)^{2}$. Now we need the following auxiliary result. Recall that $P_{\mathbf{g}}(z)=\prod_{g \in \mathbf{g}}(z+g)$.

Lemma 5. a) Let $\mathbf{g}$ be a cyclic set (perhaps, trivial). Then for any $z \in \mathbb{C}$ we have $\prod_{g \in \mathbf{g}}(z-g)=\prod_{g \in \mathbf{g}}\left(z-g^{2}\right)$.

b) Let $\mathbf{g}_{1}$ and $\mathbf{g}_{2}$ be cyclic sets (perhaps, trivial). Then

$$
\prod_{g \in \mathbf{g}_{1}} P_{\mathbf{g}_{2}}(g)= \begin{cases}1 & \text { if } \mathbf{g}_{1} \neq \mathbf{g}_{2} \\ 2^{n} & \text { if } \mathbf{g}_{1}=\mathbf{g}_{2}\end{cases}
$$

where $n=$ Card $\mathbf{g}_{1}$.

Proof. The proof is outlined in the Appendix. 
Corollary 2. Suppose $\mathbf{p}$ has a nontrivial cycle $\mathbf{g}$ and $\mathbf{p}_{\mathbf{g}}=\mathbf{p} / P_{\mathbf{g}}$; then $\mathbf{p}(1)=\mathbf{p}_{\mathbf{g}}(1)$.

Proposition 6. a) If a polynomial $\mathbf{p}$ has a symmetric zero a, then $\gamma_{\mathbf{g}}(\mathbf{p})=\gamma_{\mathbf{g}}\left(\mathbf{p}_{a}\right)$ for any cyclic set $\mathbf{g}$.

b) If $\mathbf{p}$ has a cycle $\mathbf{b}$, then for any cyclic set $\mathbf{g}$ we have $\gamma_{\mathbf{g}}(\mathbf{p})=\gamma_{\mathbf{g}}\left(\mathbf{p}_{\mathbf{b}}\right)$ if $\mathbf{g} \neq \mathbf{b}$, and $\gamma_{\mathbf{g}}(\mathbf{p})=2^{n} \gamma_{\mathbf{g}}\left(\mathbf{p}_{\mathbf{b}}\right)$ if $\mathbf{g}=\mathbf{b}$.

c) If $\mathbf{p}(z)=(z+1)^{k} \mathbf{p}_{k}(z)$, then $\gamma_{\mathbf{g}}(\mathbf{p})=\gamma_{\mathbf{g}}\left(\mathbf{p}_{k}\right)$ for any nontrivial cyclic set $\mathbf{g}$.

Proof. This follows directly from Lemma 5.

As an additional corollary, we obtain the following statement.

Proposition 7. Let $\mathbf{p}$ be an arbitrary polynomial that has a generalized cycle $\mathbf{b}=$ $\left\{b_{1}, \ldots, b_{n}\right\}$ of multiplicity $r$. Then $\gamma_{\mathbf{b}}(\mathbf{p})=2^{n r} \cdot \gamma_{\mathbf{b}}(\tilde{\mathbf{p}})$, where $\tilde{\mathbf{p}}$ is a clean polynomial.

Now we define yet another function of cyclic sets:

$$
\kappa_{\mathbf{b}}(\mathbf{p})=\left|\gamma_{\mathbf{b}}(\mathbf{p})\right|^{1 / n}=\left|\prod_{j=1}^{n} \mathbf{p}\left(b_{j}\right)\right|^{1 / n} .
$$

Lemma 6. If a polynomial $\mathbf{p}$ has no symmetric zeros but has a cycle $\mathbf{b}=\left\{b_{1}, \ldots, b_{n}\right\}$ of multiplicity $r \geq 1$, then the operators $\left.T_{i}^{*}\right|_{U_{\mathbf{b}}}$ restricted to the subspace $U_{\mathbf{b}}$ are cyclic in a suitable basis $\left\{e_{j}\right\}_{j=1}^{n}$ and act by the formula

$$
\left.T_{i}^{*}\right|_{U_{\mathbf{b}}} e_{j+1}=\frac{1}{2} \kappa_{\mathbf{b}}(\mathbf{p}) \lambda_{j i} e_{j}, \quad j=1, \ldots, n,
$$

where the modulus of each of the numbers $\lambda_{j i}$ is equal to 1.

Proof. We set $e_{j}=\left(\kappa_{\mathbf{b}}(\mathbf{p})\right)^{-j} \overline{\mathbf{p}\left(b_{1}\right)} \cdots \overline{\mathbf{p}\left(\bar{b}_{j}\right)}\left[b_{j}\right]$. Observe that $\kappa_{\mathbf{b}}(\mathbf{p}) \neq 0$; otherwise the polynomial $\mathbf{p}$ vanishes at one of the points $b_{j}$, so that $\pm b_{j}$ is a pair of symmetric zeros. It is easy to check that the basis $\left\{e_{j}\right\}_{j=1}^{n}$ is what we are looking for. It suffices to apply formulas (77) and use the fact that $\left|b_{j}\right|=1$ for all $j$.

Thus, both operators $\left.T_{i}^{*}\right|_{U_{\mathbf{b}}}$ are compositions of orthogonal operators and homotheties with positive coefficients; moreover, they have one and the same coefficient $\frac{1}{2} \kappa_{\mathbf{b}}(\mathbf{p})=$ $2^{r-1} \kappa_{\mathbf{b}}(\tilde{\mathbf{p}})$. Using Proposition [6, we obtain the following statement.

Proposition 8. Under the assumptions of Lemma 6 , the operators $T_{i}^{*}, i=0,1$, restricted to their common invariant subspace $U_{\mathbf{b}, r}$, have the form

$$
\left.T_{i}^{*}\right|_{U_{\mathbf{b}, r}}=\left(\begin{array}{cccc}
A_{\mathbf{b}, i} & * & \cdots & * \\
0 & 2^{-1} A_{\mathbf{b}, i} & * & \vdots \\
\vdots & 0 & & * \\
0 & \cdots & 0 & 2^{-(r-1)} A_{\mathbf{b}, i}
\end{array}\right), \quad i=0,1,
$$

where $A_{\mathbf{b}, 0}$ and $A_{\mathbf{b}, 1}$ are the cyclic operators on $\mathbb{R}^{n}$ defined by (19).

We see that on every subspace $U_{\mathbf{b}, r}$ the matrices of the operators $T_{i}^{*}$ in a special basis have the block upper triangular form (20). Thus, Theorem 4 characterizes all common invariant subspaces of the operators $T_{0}^{*}, T_{1}^{*}$, and Proposition 8 describes the structure of the operators on these subspaces. We arrive at the following theorem. 
Theorem 5. For any polynomial $\mathbf{p}$ of degree $N$, there is a basis in $\mathbb{R}^{N}$ for which the matrices of the operators $T_{0}, T_{1}$ have a block lower triangular form,

$$
T_{i}=\left(\begin{array}{ccccccccc}
0 & 0 & \cdots & 0 & \cdots & \cdots & \cdots & \cdots & 0 \\
* & 0 & \cdots & 0 & \cdots & \cdots & \cdots & \cdots & 0 \\
\vdots & * & & 0 & \vdots & \vdots & \vdots & \vdots & 0 \\
* & \cdots & * & 0 & 0 & \vdots & \vdots & \vdots & 0 \\
* & & & * & B_{0, i} & 0 & \vdots & \vdots & 0 \\
\vdots & & & \vdots & * & B_{1, i} & 0 & \vdots & 0 \\
\vdots & & & \vdots & \vdots & * & & 0 & \vdots \\
* & & & * & * & \cdots & * & B_{s, i} & 0 \\
* & \cdots & \cdots & * & * & \cdots & * & * & \tilde{T}_{i}
\end{array}\right), \quad i=0,1
$$

where the matrices $B_{k, i}$ are adjoint to the matrices (20), the $\mathbf{b}_{k}$ are generalized cycles of $\mathbf{p}$ of multiplicities $r_{k}, k=0, \ldots, s$, and the $\tilde{T}_{i}$ are the matrices of the clean polynomial $\tilde{\mathbf{p}}$.

The following case is most important and well studied: $\mathbf{p}$ has neither symmetric zeros nor nontrivial cycles, but has a trivial cycle of some multiplicity $r \geq 1$. In this case $\mathbf{p}(z)=(z+1)^{r} \tilde{\mathbf{p}}(z)$.

Proposition 9. If the polynomial $\mathbf{p}$ has neither symmetric zeros nor nontrivial cycles, then the polynomials $T_{0}, T_{1}$ have a chain of common invariant subspaces

$$
G_{r} \subset \cdots \subset G_{0}=\mathbb{R}^{N}
$$

where $r$ is the multiplicity of the root $z=-1$ of $\mathbf{p}$. The subspace $G_{j}$ has dimension $N-j$ and is the orthogonal complement to the subspace

$$
G_{j}^{\perp}=\operatorname{span}\left\{\left(1^{k}, 2^{k}, \ldots, N^{k}\right)^{T}, k=0, \ldots, j-1\right\} .
$$

There are no other common invariant subspaces of $T_{0}, T_{1}$. The matrices of these operators in an appropriate basis look like this:

$$
T_{i}=\left(\begin{array}{cccccccc}
\frac{1}{2} \mathbf{p}(1) & 0 & 0 & \cdots & 0 & 0 & \cdots & 0 \\
* & \frac{1}{2^{2}} \mathbf{p}(1) & 0 & \cdots & 0 & 0 & \cdots & 0 \\
* & * & \frac{1}{2^{3}} \mathbf{p}(1) & \cdots & 0 & 0 & \cdots & 0 \\
\vdots & \vdots & & & 0 & 0 & \cdots & 0 \\
* & * & * & \cdots & \frac{1}{2^{r}} \mathbf{p}(1) & 0 & \cdots & 0 \\
* & * & * & \cdots & * & & & \\
\vdots & & & & \vdots & \tilde{T}_{i} & \\
* & * & * & \cdots & * & &
\end{array}\right), \quad i=0,1,
$$

where the $\tilde{T}_{i}$ are the matrices corresponding to the clean polynomial $\tilde{\mathbf{p}}$.

Proof. Under these assumptions, the polynomial has only a trivial cycle of multiplicity $r$. Using Corollary 1 and applying (16) to the trivial cycle $\mathbf{b}=\{1\}$, we complete the proof.

Corollary 3. Under the assumptions of Proposition 9 , every common invariant subspace of the operators $T_{0}, T_{1}$ contains the subspace $G_{r}$.

Remark 1. Factorizations of the type (23) originated in 4]; then they appeared in the literature many times. They always dealt with the special case where the polynomial has 
neither symmetric zeros nor nontrivial cycles. We add one new result to this special case: the operators $T_{0}, T_{1}$ have no common invariant subspaces other than those in the chain $\left\{G_{j}\right\}$. We shall need this fact in the next chapter for the study of refinement equations.

\section{Chapter II. Applications to Refinement equations}

\section{§7. Cleaning of Refinement equations}

For arbitrary coefficients $c_{0}, \ldots, c_{N}$ such that $\sum c_{k}=2, c_{0} c_{N} \neq 0$, we consider the refinement equation (3). It always possesses a unique compactly supported solution $\varphi$ (in the space of tempered distributions) normalized by the condition $\int \varphi=1$. This solution, which is called a refinable function, has its support on the segment $[0, N]$. The main problem is to determine, knowing the coefficients $\left\{c_{k}\right\}$ of the equation, whether $\varphi$ belongs to the spaces $L_{p}(\mathbb{R})$ or $C(\mathbb{R})$, and what is the regularity of $\varphi$ in these spaces.

The sequence $\left\{c_{k}\right\}$ is usually called the mask of the equation; the polynomial $\mathbf{m}(z)=$ $\frac{1}{2} \sum_{k=0}^{N} c_{k} z^{k}$ is called the symbol. The symbol is half the generating polynomial (2). For some reason, all publications on refinement equations deal with the symbol rather than the generating polynomial. We shall also obey this rule. The results of Chapter I can easily be rewritten in terms of the symbol $\mathbf{m}$. The results of $\S \S 2-5$ remain the same (since $\mathbf{m}$ and $\mathbf{p}$ have the same roots); in $\S 6$ we can use the relations $\kappa_{\mathbf{b}}(\mathbf{m})=\frac{1}{2} \kappa_{\mathbf{b}}(\mathbf{p})$ and $\gamma_{\mathbf{b}}(\mathbf{m})=2^{-n} \kappa_{\mathbf{b}}(\mathbf{p})$. The condition $\sum_{k} c_{k}=2$ implies that $\mathbf{m}(1)=1$. A necessary, but not sufficient, condition for the smoothness of refinable functions was derived in [9] for $l=0$ and in 24] for all $l$.

Lemma 7. If $\varphi \in W_{1}^{l}$ for some $l \geq 0$, then the symbol possesses a trivial generalized cycle of multiplicity at least $l+1$.

Thus, a refinement equation admitting a smooth solution can always be cleaned out of symmetric zeros and cycles $(\S 3)$. How does this cleaning change the solution $\varphi$ ? The elimination of a symmetric zero implies passage to a new equation with the symbol $\mathbf{m}_{a}(z)=\frac{z-a}{z^{2}-a} \mathbf{m}(z)$. Since $\mathbf{m}_{a}(1)=\mathbf{m}(1)=1$, the new equation has a compactly supported solution, which will be denoted by $\varphi_{a}$. The degree of the polynomial is reduced by 1 , so that $\operatorname{supp} \varphi_{a}=[0, N-1]$.

Removing the trivial cycle results in the polynomial $\mathbf{m}_{1}(z)=\frac{1}{z+1} \mathbf{m}(z)$. We have $\mathbf{m}_{1}(1)=\frac{1}{2}$, although at the point $z=1$, the symbol should take the value 1 , but not $\frac{1}{2}$. That is why the removal of the trivial cycle leads to the equation with symbol $\mathbf{m}_{1}$, but not with $2 \mathbf{m}_{1}$. Now we have $2 \mathbf{m}_{1}(1)=1$, and in the space of distributions the equation has a solution $\varphi_{1}$ with $\operatorname{supp} \varphi_{1} \subset[0, N-1]$. Finally, if we remove a nontrivial cycle $\mathbf{b}=\left\{b_{1}, \ldots, b_{n}\right\}(n \geq 2)$, then we pass to the polynomial $\mathbf{m}_{\mathbf{b}}(z)=\mathbf{m}(z) / P_{\mathbf{b}}(z)$. Since $\mathbf{m}_{\mathbf{b}}(1)=\mathbf{m}(1)$ by Corollary 2 , it follows that the new equation has a compactly supported solution $\varphi_{\mathbf{b}}$, with $\operatorname{supp} \varphi_{\mathbf{b}} \subset[0, N-n]$. As a result, we obtain a clean symbol $\tilde{\mathbf{m}}$ and a refinable function $\tilde{\varphi}$. Lemma 7 implies that $\tilde{\varphi} \notin L_{1}$, which means that the function $\tilde{\varphi}$ is purely singular.

Proposition 10. If we remove a pair of symmetric zeros $\pm \sqrt{a}$, then

$$
\varphi(\cdot)=a \varphi_{a}(\cdot)-\varphi_{a}(\cdot-1)
$$

if we remove a nontrivial cycle $\mathbf{b}$, then

$$
\varphi(\cdot)=\sum_{k=0}^{n} q_{k} \varphi_{\mathbf{b}}(\cdot-k)
$$


where $\left\{q_{k}\right\}$ are the coefficients of the polynomial $Q_{\mathbf{b}}$ given by (10); if we remove the trivial cycle, then

$$
\varphi=\varphi_{1} * \chi_{[0,1)}
$$

Proof. See the Appendix.

Remark 2. It would be more proper to normalize the function $\varphi$ in (24) by the coefficient $1 /(a-1)$ to ensure the condition $\widehat{\varphi}(0)=1$. We avoid doing this for simplicity.

Now we need to introduce operators $\mathcal{D}_{a}, \mathcal{D}_{\mathbf{b}}$ in the space of tempered distributions. The operator $\mathcal{D}_{a}$ depends on a complex parameter $a \in \mathbb{C}$ and acts by the formula

$$
\left[\mathcal{D}_{a} f\right](\cdot)=a f(\cdot)-f(\cdot-1) ;
$$

the operator $\mathcal{D}_{\mathbf{b}}$ corresponds to a cyclic set $\mathbf{b}$ and is defined by

$$
\left[\mathcal{D}_{\mathbf{b}} f\right](\cdot)=\sum_{k=0}^{n} q_{k} f(\cdot-k),
$$

where $\left\{q_{k}\right\}$ are the coefficients of the polynomial $Q_{\mathbf{b}}$ given by (10). Relations (24) and (25) can now be written as $\varphi=\mathcal{D}_{a} \varphi_{a}, \varphi=\mathcal{D}_{\mathbf{b}} \varphi_{\mathbf{b}}$. Proposition 10 reduces any refinement equation to that with a clean symbol. Moreover, this reduction controls the regularity of solutions. From (24) and (25) it follows that $\varphi$ is at least as regular as $\varphi_{a}$ or $\varphi_{\mathbf{b}}$. On the other hand, it is easy to deduce formulas inverse to (24) and (25). For example, $\varphi_{a}(x)=\left[\mathcal{D}_{a}^{-1} \varphi\right](x)=\sum_{k=0}^{\infty} a^{k} \varphi(x-k)$. This shows that $\varphi_{a}$ and $\varphi_{\mathbf{b}}$ are at least as regular as $\varphi$. Thus, the removal of symmetric zeros and nondegenerate cycles respects the regularity of refinable functions. The removal of a trivial cycle reduces regularity by one. Indeed, the convolution with the function $\chi_{[0,1)}$ (see formula (26)) is spanned by the integer translates of the primitive $\int_{-\infty}^{x} \varphi_{1}(t) d t$.

A refinable function is said to be stable if its integer translates $\{\varphi(\cdot-k), k \in \mathbb{Z}\}$ are linearly independent. Stability is equivalent to the absence of symmetric zeros and nontrivial cycles (see [25, Theorem 3.4.10]).

Suppose that the cleaning algorithm removes consecutively the symmetric zeros $a_{1}, \ldots$, $a_{t}$ and the cycles $\mathbf{b}_{0}, \ldots, \mathbf{b}_{s}$ of multiplicities $r_{0}, \ldots, r_{s}$, respectively. We recall that $\mathbf{b}_{0}=$ $\{1\}$ is a trivial cycle, and the other cycles are nontrivial. We have

$$
\varphi=B_{r_{0}-1} * \mathcal{D}_{\mathbf{b}_{s}}^{r_{s}} \cdots \mathcal{D}_{\mathbf{b}_{1}}^{r_{1}} \mathcal{D}_{a_{t}} \cdots \mathcal{D}_{a_{1}} \tilde{\varphi}
$$

where $r_{j}$ is the multiplicity of $\mathbf{b}_{j}$, the difference operators $\mathcal{D}_{a_{i}}$ and $\mathcal{D}_{\mathbf{b}_{i}}$ are defined in (27) and (28), and $B_{r}$ is a cardinal $B$-spline of order $r$. All the operators $\mathcal{D}_{a_{i}}, \mathcal{D}_{\mathbf{b}_{i}}$ commute with each other and with the convolution operator. Hence, the factors in (29) can be grouped in several different ways, which leads to several representations of the refinable function.

Theorem 6 (Factorization theorem). a) An arbitrary refinable function $\varphi$ can be represented in a unique way as the convolution of a clean refinable function $\tilde{\varphi}$ with a spline with integral nodes:

$$
\varphi=S_{r_{0}-1} * \tilde{\varphi}, \quad \text { where } S_{r_{0}-1}=\mathcal{D}_{\mathbf{b}_{s}}^{r_{s}} \cdots \mathcal{D}_{\mathbf{b}_{1}}^{r_{1}} \mathcal{D}_{a_{t}} \cdots \mathcal{D}_{a_{1}} B_{r_{0}-1} .
$$

b) If $\varphi \in C^{l}$ or $\varphi \in W_{p}^{l}(l \geq 1)$, then $\varphi$ can be represented in a unique way as the convolution of a stable continuous (or, respectively, $L_{p}$ ) refinable function with a spline of order $l-1$ with integral nodes:

$$
\varphi=S_{l-1} * \varphi_{l}, \quad \text { where } \varphi_{l}=B_{r_{0}-l-1} * \tilde{\varphi}, S_{l-1}=\mathcal{D}_{\mathbf{b}_{s}}^{r_{s}} \cdots \mathcal{D}_{\mathbf{b}_{1}}^{r_{1}} \mathcal{D}_{a_{t}} \cdots \mathcal{D}_{a_{1}} B_{l-1} .
$$

Moreover, $\alpha_{\varphi}=\alpha_{\varphi_{l}}+l, \alpha_{\varphi, p}=\alpha_{\varphi_{l}, p}+l, p \geq 1$. 
Theorem 6 completely reduces the study of smooth refinable functions to $L_{p}$-functions (to continuous functions if $p=\infty$ ). This enables us to assume in the next section that $\varphi \notin W_{p}^{1}$, and accordingly, $\alpha_{\varphi, p}=\sup \left\{\alpha \geq 0, \omega_{p}(\varphi, h) \leq C h^{\alpha}\right\}$.

\section{$\S 8$. The Space $V_{\varphi}$ And the operators $T_{0}, T_{1}$ ON IT}

The geometric approach for computing the regularity of refinable functions was developed in [3, 4, 18. The crucial idea is to pass from $\varphi$ to the vector-valued function

$$
v_{f}(x)=(f(x), \ldots, f(x-N+1))^{T} \in \mathbb{R}^{N}, \quad x \in[0,1] .
$$

The refinement equation (3) becomes

$$
v(x)=T_{i} v(2 x-i), \quad x \in\left[\frac{i}{2}, \frac{i+1}{2}\right], \quad i=0,1,
$$

where $v=v_{\varphi}$ and $T_{0}, T_{1}$ are the Toeplitz operators (11) of the sequence $\left\{c_{0}, \ldots, c_{N}\right\}$. Consider the following two subspaces in $\mathbb{R}^{N}$ :

$$
V_{\varphi}=\operatorname{span}\{v(x)-v(y), x, y \in[0,1]\}, \quad \bar{V}_{\varphi}=\operatorname{span}\{0, v(x), x \in[0,1]\},
$$

where both identities are fulfilled almost everywhere. In the sequel we omit the subscript $\varphi$ if the function $\varphi$ that we mean is clear.

In [5, 19] it was shown that a refinable function $\varphi$ is in $L_{p}$ (or in $C$ if $p=\infty$ ) provided $\rho_{p}\left(\left.T_{0}\right|_{V},\left.T_{0}\right|_{V}\right)<1$. Furthermore,

$$
\alpha_{\varphi, p}=-\log _{2} \rho_{p}\left(\left.T_{0}\right|_{V},\left.T_{0}\right|_{V}\right)
$$

where $\rho_{p}$ is the joint spectral radius (4). Recall that we focus on the case where $\varphi \notin W_{p}^{1}$. A slightly sharper result was obtained in [13:

$$
\omega_{p}(\varphi, h) \asymp\left\|\Sigma_{k}(u)\right\|_{p}, \quad k=\left[-\log _{2} h\right],
$$

where

$$
\left\|\Sigma_{k}(u)\right\|_{p}=\left(2^{-k} \sum_{d_{1}, \ldots, d_{k} \in\{0,1\}}\left\|T_{d_{1}} \cdots T_{d_{k}}(u)\right\|^{p}\right)^{1 / p}
$$

(with the usual modifications for $p=\infty$ ), and the vector $u$ is equal to $\left(T_{1}-T_{0}\right) u_{0}$ with $u_{0}=\int_{0}^{1} v(x) d x$. Relation (32) provides a way, which is unique thus far, to obtain sharp values of the exponents of regularity for refinable functions. Its implementations, however, are complicated by the fact that the space $V$ is unknown initially. Usually, $V$ is found as the smallest (by inclusion) common invariant subspace of $T_{0}, T_{1}$ that contains the vector $u$ [5, 19]. Similarly, $\bar{V}$ is the smallest common invariant subspace containing $u_{0}$. Obviously, $\bar{V}=\operatorname{span}\left\{u_{0}, V\right\}$, and $u_{0}$ is an eigenvector of $\frac{1}{2}\left(T_{0}+T_{1}\right)$ that corresponds to the eigenvalue 1 . However, the eigenvalue 1 may be multiple. In this case it is not clear how to find the required vector $u_{0}$ in the corresponding root subspace. We discuss this issue in more detail in $\S 15$. Even in the case of a simple eigenvalue, the definitions of the subspaces $\bar{V}$ and $V$ tell us nothing about their structure and dimensions or about the operators $\left.T_{i}\right|_{V}$. It is natural to ask whether explicit formulas for those subspaces and for those operators exist, which are suitable for all refinement equations. In various reformulations, this problem was studied in [3]-7] and in [9, 10]. In this section we obtain its complete solution (see Theorems 7 and 8 ).

We start with some comments. First, $V$ and $\bar{V}$ cannot coincide. Indeed, $\rho_{1}\left(\left.T_{0}\right|_{V},\left.T_{1}\right|_{V}\right)$ $<1$ because $\varphi \in L_{1}$, but $\rho_{1}\left(\left.T_{0}\right|_{\bar{V}},\left.T_{1}\right|_{\bar{V}}\right) \geq 1$, because the operator $\frac{1}{2}\left(T_{0}+T_{1}\right)$ restricted to $\bar{V}$ possesses the eigenvalue 1 . Thus, the dimension of $V$ is less by one than that of $\bar{V}$. Recall that $E_{q}$ denotes the largest root subspace of the operators $T_{0}^{*}, T_{1}^{*}$; this subspace can be found with the help of Proposition 5. By $U_{\mathbf{b}, k}$ we denote the common invariant subspaces of the operators $T_{i}^{*}$ defined by (16). For given $\mathbf{b}$, these subspaces form an 
embedded system $U_{\mathbf{b}}=U_{\mathbf{b}, 1} \subset \cdots \subset U_{\mathbf{b}, r_{\mathbf{b}}}$. We denote by $\mathbf{b}_{0}$ the trivial generalized cycle.

Theorem 7. The space $\bar{V}$ is the orthogonal complement to the linear span of $E_{q}$ and the spaces $U_{\mathbf{b}, r}$ taken for all nontrivial generalized cycles $\mathbf{b}$ of the symbol, $r=r_{\mathbf{b}}$. The space $V$ is the orthogonal complement to the linear span of the same spaces and $U_{\mathbf{b}_{0}, 1}$.

Proof. Since $\bar{V}$ is the smallest common invariant subspace of $T_{0}, T_{1}$ containing $u_{0}$, relation (15) shows that $\bar{V}$ is orthogonal to $E_{q}$. Now, in the block lower triangular factorization (21), we find the indecomposable blocks (19) contained in the subspace $\bar{V}$. Since $\frac{1}{2}\left(T_{0}+T_{1}\right) u_{0}=u_{0}$, it follows that these blocks have eigenvalue 1 . However, $V$ cannot contain such blocks, because the radius $\rho_{1}$ of the operators $T_{0}, T_{1}$ is smaller than 1 on this subspace. But since $\operatorname{dim} \bar{V}-\operatorname{dim} V=1$, we see that $\bar{V}$ can contain only the block corresponding to the trivial generalized cycle. The dimensions of the other blocks are at least two. Hence, $\bar{V}$ is orthogonal to the subspaces $U_{\mathbf{b}, r}$ for all nontrivial $\mathbf{b}$. The last block is of the form (23), where $\frac{1}{2} \mathbf{p}(1)=\mathbf{m}(1)=1$; it is contained in $\bar{V}$ entirely. Otherwise, the diagonal does not contain the first element $\frac{1}{2} \mathbf{p}(1)=1$, and therefore, the matrix $\frac{1}{2}\left(\tilde{T}_{0}+\tilde{T}_{1}\right)$ has the eigenvalue 1 . But in this case the first $r_{0}$ entries of the corresponding eigenvector $u_{0}$ are zero, i.e., $u_{0} \in \tilde{V}$, where $\tilde{V}$ is the subspace corresponding to the block $\tilde{T}_{i}$. Consequently, $V \subset \bar{V} \subset \tilde{V}$. Since the operators $\tilde{T}_{i}$ have no common invariant subspaces (see Theorem [1), we have $V=\bar{V}=\tilde{V}$. This is impossible, because $V$ and $\bar{V}$ have different dimensions.

In practice the space $V$ and the operators $\left.T_{i}\right|_{V}$ can be found with the help of the cleaning algorithm. Recall that the symmetric zeros are deleted first, then the nontrivial cycles follow, and finally the trivial one. Assume that all symmetric zeros and nontrivial cycles are already removed, with their multiplicities. Then we remove exactly one trivial cycle and obtain some polynomial $\mathbf{m}_{r}$. The symbol $\mathbf{m}_{r}$ has neither symmetric zeros nor nontrivial cycles, but it has a trivial cycle of multiplicity $r-1$. The corresponding transfer matrix is

$$
C_{r}=C_{\mathbf{b}_{0}} \cdot C_{\mathbf{b}_{s}, r_{s}} \cdots C_{\mathbf{b}_{s}, 1} \cdot C_{\mathbf{b}_{s-1}, r_{s-1}} \cdots C_{\mathbf{b}_{s-1}, 1} \cdots C_{\mathbf{b}_{1}, r_{1}} \cdots C_{\mathbf{b}_{1}, 1} C_{a_{t}} \cdots C_{a_{1}} .
$$

Applying Theorem [7, we arrive at the following statement.

Proposition 11. If $\varphi \in L_{1}$, then the subspace $V$ is spanned by the columns of the matrix $C_{r}$, and the restrictions of $T_{0}, T_{1}$ to $V$ are isomorphic to the operators of the symbol $\mathbf{m}_{r}$.

Remark 3. The space $V$ appears after elimination of all symmetric zeros and of one trivial cycle. In particular, if the symbol has no symmetric zeros at all, then $\bar{V}=\mathbb{R}^{N}$ and $V=U_{1}^{\perp}=\left\{x \in \mathbb{R}^{N}, \sum_{k} x_{k}=0\right\}$.

Remark 4. Proposition 11 makes it possible to determine the spaces $\bar{V}$ and $V$ for any refinement equation, not only for those having integrable solutions. It is only required that the symbol possess a trivial generalized cycle. The subspace $V$ is spanned by the columns of the matrix $C_{r}$ defined by (34). The corresponding operator maps $\mathbb{R}^{N}$ onto $V$ surjectively.

Thus, the operators $T_{0}, T_{1}$ restricted to $V$ are isomorphic to the pair of operators corresponding to the symbol $\mathbf{m}_{L}$. Since the symbol $\mathbf{m}_{L}$ has neither symmetric zeros nor nontrivial cycles, we can apply Proposition 9. This proves the following theorem on the structure of Toeplitz operators on the subspace $V$.

Theorem 8. Assume that the symbol of a refinement equation has a trivial generalized cycle of multiplicity $r \geq 1$. Then the restrictions of the operators $T_{0}, T_{1}$ to $V$ are isomorphic to the Toeplitz operators that correspond to the symbol $\mathbf{m}_{r}$ obtained from $\mathbf{m}$ by 
removing all symmetric zeros, all nontrivial cycles, and one trivial cycle. This isomorphism is determined by the transfer matrix (34). In a suitable basis, the matrices of the operators $\left.T_{i}\right|_{V}, i=0,1$, have the following form: if $r=1$, then $\left.T_{i}\right|_{V}=\tilde{T}_{i}$; if $r \geq 2$, then

$$
\left.T_{i}\right|_{V}=\left(\begin{array}{cccccccc}
2^{-1} & 0 & 0 & \cdots & 0 & 0 & \cdots & 0 \\
* & 2^{-2} & 0 & \cdots & 0 & 0 & \cdots & 0 \\
* & * & 2^{-3} & \cdots & 0 & 0 & \cdots & 0 \\
\vdots & \vdots & & & 0 & 0 & \cdots & 0 \\
* & * & * & \cdots & 2^{-r+1} & 0 & \cdots & 0 \\
* & * & * & \cdots & * & & & \\
\vdots & & & & \vdots & & \tilde{T}_{i} \\
* & * & * & \cdots & * & &
\end{array}\right), \quad i=0,1
$$

where the $\tilde{T}_{i}$ are the matrices of the clean symbol $\tilde{\mathbf{m}}$.

The next statement is true because the operators $\tilde{T}_{i}$ are nondegenerate (see Theorem 11).

Corollary 4. Both operators $T_{i}$ are nondegenerate on the space $V$.

Applying Proposition 9 to the operators (35), we get the following.

Corollary 5. All nontrivial common invariant subspaces of the operators (35) belong to the chain

$$
G_{j}=\left\{x \in \mathbb{R}^{N}, x_{1}=\cdots=x_{j}=0\right\} .
$$

Corollary 6. If the symbol of a refinement equation has neither symmetric zeros nor nontrivial cycles, then

$$
V=W=\left\{x \in \mathbb{R}^{N}, \sum x_{k}=0\right\}
$$

\section{§9. Regularity of REFinable FUnCtions}

Formula (32) expresses the regularity of a refinable function in terms of the $p$-radius of the operators $T_{0}, T_{1}$ restricted to the subspace $V$. Theorem 8 reduces the problem to the operators $\tilde{T}_{0}, \tilde{T}_{0}$ of the clean symbol. The main advantage is that the explicit form of these operators is known. Moreover, as a rule, the dimension of the operators $\tilde{T}_{i}$ is smaller than that of $\left.T_{i}\right|_{V}$. This is important, because computation of the joint spectral radius becomes much more difficult when the dimension grows.

We need some auxiliary results. Any pair of linear operators $A_{0}, A_{1}$ has, in a suitable basis, the block lower triangular form:

$$
A_{i}=\left(\begin{array}{ccccc}
A_{i}^{1} & 0 & 0 & \cdots & 0 \\
* & A_{i}^{2} & 0 & \cdots & 0 \\
* & * & * & & \vdots \\
\vdots & & & & 0 \\
* & \cdots & \ldots & * & A_{i}^{l}
\end{array}\right), \quad i=0,1
$$

where each pair $\left\{A_{0}^{k}, A_{1}^{k}\right\}$ is irreducible. We set $\rho_{p, k}=\rho_{p}\left(B_{0}^{k}, B_{1}^{k}\right)$ and consider the $v a$ lency of the pair $A_{0}, A_{1}$, i.e., the number of pairs $\left\{A_{0}^{k}, A_{1}^{k}\right\}$ for which $\rho_{p, k}=\max _{j=1, \ldots, l} \rho_{p, j}$. The valency is a discrete function of the parameter $p$. The number $l$ of blocks and their dimensions are defined uniquely up to permutations; the matrices $A_{i}^{k}$ are defined uniquely up to similarities (transfers to other bases in $\mathbb{R}^{N}$ ). Hence, the values $\rho_{p, j}$ and the valency $s$ are well defined for any operators and any $p \in[1,+\infty]$. 
Lemma 8. For any operators $A_{0}, A_{1}$ and any $p \in[1,+\infty]$ we have $\rho_{p}=\max _{j=1, \ldots, l} \rho_{p, j}$.

For an arbitrary vector $u \in \mathbb{R}^{N}$ we denote by $V_{u}$ the smallest (by inclusion) common invariant linear subspace of $A_{0}, A_{1}$ that contains $u$.

Proposition 12. For any operators $A_{i}, i=0,1$, any $p \in[1,+\infty]$, and any $u \in \mathbb{R}^{N}$ with $\|u\|=1$, there are positive constants $c_{1}, c_{2}$ such that

$$
c_{1}\left(\rho_{p}\right)^{k} \leq\left\|\Sigma_{k}(u)\right\|_{p} \leq c_{2} k^{s-1}\left(\rho_{p}\right)^{k}, \quad k \in \mathbb{N},
$$

where $\rho_{p}$ is the p-radius of the operators $\left.A_{0}\right|_{V_{u}},\left.A_{1}\right|_{V_{u}}$, and $s$ is their valency.

The proofs of Lemma 8 and Proposition 12 can be found in [13].

Now we are able to introduce the main results on the regularity of refinable functions. Suppose the symbol $\mathbf{m}$ of a given equation has a trivial cycle of multiplicity $r_{0}$. Let $\tilde{\mathbf{m}}$ be the corresponding clean symbol, let $\tilde{T}_{0}, \tilde{T}_{1}$ be the Toeplitz operators of $\tilde{\mathbf{m}}$, and let $\rho_{p}=\rho_{p}\left(\tilde{T}_{0}, \tilde{T}_{1}\right), p \in[1,+\infty]$.

To compute the joint spectral radii of the Toeplitz operators, we invoke the results of Chapter I. By Lemma 6, if a polynomial has a cycle $\mathbf{b}$, then

$$
\left.T_{d_{1}}^{*} \cdots T_{d_{n}}^{*}\right|_{U_{\mathbf{b}}}=\gamma_{\mathbf{b}}(\mathbf{m}) \cdots \Lambda_{d_{1} \cdots d_{n}}, \quad d_{1}, \ldots, d_{n} \in\{0,1\},
$$

where the matrix $\Lambda_{d_{1} \cdots d_{n}}$ is diagonal with the moduli of all diagonal elements equal to 1. Therefore, all $p$-radii $\rho_{p}$ of these operators are the same. Indeed,

$$
\rho_{p}\left(\left.T_{0}^{*}\right|_{U_{\mathbf{b}}},\left.T_{1}^{*}\right|_{U_{\mathbf{b}}}\right)=\kappa_{\mathbf{b}}(\mathbf{m})=2^{r} \kappa_{\mathbf{b}}(\tilde{\mathbf{m}}), \quad p \in[1,+\infty],
$$

where $\kappa_{\mathbf{b}}(\mathbf{m})$ is the quantity defined in (18). Using Lemma 8 , we see that the $p$-radii of the block matrices (20) associated with a generalized cycle $\mathbf{b}$ are equal to $\kappa_{\mathbf{b}}(\mathbf{m})$ for all $p$, and the valency is 1 . Now formula (21) allows us to express the $p$-radii of any Toeplitz operators in terms of those of the Toeplitz operators $\tilde{T}_{i}$ of the clean symbol. We denote $\rho_{p}=\rho_{p}\left(\tilde{T}_{0}, \tilde{T}_{1}\right)$.

Theorem 9. A refinable function $\varphi$ belongs to $W_{p}^{k}(k \geq 0)$ if and only if $\rho_{p}<2^{-k}$. Moreover, $\alpha_{\varphi, p}=-\log _{2} \rho_{p}$. In case $p=\infty$, the space $W_{p}^{k}$ is replaced with $C^{k}$.

Proof. Let $l \geq 0$ be the largest integer for which $\varphi \in W_{p}^{l}$. We may assume that $l=0$, because otherwise we can use Theorem $[6$ on factorization to write $\varphi$ as the convolution $B_{l-1} * \varphi_{l}$ and pass to the function $\varphi_{l}$. Then $\alpha_{\varphi, p}=-\log _{2} \rho_{p}\left(\left.T_{0}\right|_{V},\left.T_{1}\right|_{V}\right)$. By Theorem 8 , the matrices of the operators $\left.T_{i}\right|_{V}$ are of the block form (35). Applying Lemma 8 to them, we get

$$
\rho_{p}\left(\left.T_{0}\right|_{V},\left.T_{1}\right|_{V}\right)=\max \left\{2^{-1}, \ldots, 2^{-r+1}, \rho_{p}\right\} .
$$

If $\rho_{p}<2^{-1}$, then, by Proposition 10, the function $\varphi_{1}(x)=\varphi^{\prime}(x)-\varphi^{\prime}(x-1)$ satisfies the refinement equation with the symbol $\mathbf{m}_{1}(z)=\frac{2}{z+1} \mathbf{m}(z)$. The Toeplitz operators of $\mathbf{m}_{1}$ restricted to the corresponding subspace $V_{1}$ have the form (35) with the blocks $2^{-1}, \ldots, 2^{-r+2}, 2 \tilde{T}_{i}$. Therefore, their radius $\rho_{p}$ is $\max \left\{2^{-1}, \ldots, 2^{-r+2}, 2 \tilde{T}_{i}\right\}<1$, because $\rho_{p}\left(2 \tilde{T}_{0}, 2 \tilde{T}_{1}\right)=2 \rho_{p}<1$. Then (32) implies that $\varphi_{1} \in L_{p}$, whence $\varphi \in W_{p}^{1}(\mathbb{R})$, which contradicts the assumption $l=0$. Thus, $\rho_{p} \geq 2^{-1}$. Combined with (40), this yields $\rho_{p}\left(\left.T_{0}\right|_{V},\left.T_{1}\right|_{V}\right)=\rho_{p}\left(\tilde{T}_{0}, \tilde{T}_{1}\right)$, which concludes the proof.

Theorem 10. Let $p \in[1,+\infty]$, and let $\varphi \in L_{p}$ be a refinable function $(\varphi \in C$ if $p=\infty)$. If the exponent $\alpha_{\varphi, p}$ is not an integer, then

$$
\omega\left(\varphi^{(l)}, h\right)_{p} \asymp h^{\alpha_{\varphi, p}-l}, \quad h \rightarrow 0,
$$


where $l=\left[\alpha_{\varphi, p}\right]$. If $\alpha_{\varphi, p}=l+1$ is an integer, then

$$
C_{1} h \leq \omega\left(\varphi^{(l)}, h\right)_{p} \leq C_{2} h|\ln h|,
$$

where $C_{1}, C_{2}$ are positive constants.

Proof. The factorization theorem (Theorem 6) allows us to assume that $l=0$. By (33), we have $\omega_{p}(\varphi, h) \asymp\left\|\Sigma_{k}(u)\right\|_{p}$, where $u=\int_{0}^{1} v(t) d t$, and $V_{u}$ is the smallest common invariant subspace of $T_{0}, T_{1}$ containing $u$. Therefore, $V_{u}=V$, and the operators $T_{i}$ have the block form (35) on $V$. If $\rho_{p}\left(\tilde{T}_{0}, \tilde{T}_{1}\right)=2^{-j}$ for some $j \in\{1, \ldots, r-1\}$ (by Theorem 9, this means that the regularity exponent $\alpha_{\varphi}$ is an integer), then the valency $s$ is 2 ; otherwise this is 1 . It remains to apply Proposition 12

\section{Chapter III. Applications to subdivision schemes and Cascade Algorithms}

\section{$\S 10$. Convergence CRITERION}

Let $\ell, \ell_{\infty}$, and $\ell_{p}$ denote (respectively) the space of all sequences $\left\{\lambda_{k}\right\}_{k=-\infty}^{+\infty}$, the space of uniformly bounded sequences, and the space of sequences summable with power $p$. For an arbitrary sequence $\left\{c_{k}\right\}_{k=0}^{N}$, the subdivision operator $B$ acts on the space $\ell$ by the formula

$$
[B \lambda]_{j}=\sum_{k} c_{j-2 k} \lambda_{k}, \quad \lambda \in \ell .
$$

A subdivision scheme (algorithm) constructs the sequence of iterations of the operator $B$ applied to a given initial sequence $\lambda \in \ell$. A subdivision scheme converges in $L_{p}$ for given $p \in[1,+\infty]$ if for any $\lambda \in \ell_{p}$ there is a function $f \in L_{p}(\mathbb{R})$ such that

$$
\left\|f(\cdot)-\sum_{k \in \mathbb{Z}}\left[B^{n} \lambda\right]_{k} \chi_{[0,1)}\left(2^{n} \cdot-k\right)\right\|_{p} \rightarrow 0, \quad n \rightarrow \infty .
$$

As usual, for $p=\infty$ we consider the space $C$. The subdivision schemes are natural extensions of the well-known de Rham and Chaikin algorithms of corner cutting. They are used in approximation theory, interpolation, curve design, etc. (see [1, 2, 3, 26] and the references therein). If a subdivision scheme converges in $L_{p}$, then the refinement equation with the same coefficients possesses a solution in $L_{p}$ (in $C$ if $p=\infty$ ), and the corresponding symbol satisfies $\mathbf{m}(-1)=0$ or, equivalently,

$$
\sum_{k} c_{2 k}=\sum_{k} c_{2 k+1}=1 \text {. }
$$

The proof can be found, e.g., in 27.

The transition operator $T$ is defined on the space of compactly supported tempered distributions by the formula

$$
[T f](x)=\sum_{k=0}^{N} c_{k} f(2 x-k) .
$$

A cascade algorithm is the process of consecutive approximations of the refinable function $\varphi$ by iterations of the operator $T$ applied to a given initial function $f(x)$. For any $f$ such that $\int f d x=1$, as $n \rightarrow \infty$ the sequence $T^{n} f$ weakly converges to $\varphi$ in the space of distributions $\mathcal{S}^{\prime}$ (see [25, Theorem 2.4.4]). The main problem is to analyze this convergence in a stronger sense, say, in the space $C(\mathbb{R})$ or in $L_{p}(\mathbb{R})$. A cascade algorithm converges in $L_{p}$ if

$$
\left\|T^{n} f-\varphi\right\|_{p} \rightarrow 0, \quad n \rightarrow 0
$$


for any compactly supported function $f \in L_{p}$ such that $\sum_{k} f(x-k) \equiv 1$ a.e. The convergence of a cascade algorithm is equivalent to that of the subdivision scheme with the same coefficients (see [3, 27]). Cascade algorithms are applied in the numerical approximation of refinable functions and wavelets and also in fast algorithms of wavelet expansions [2, 14].

Thus, the following two conditions are necessary for the convergence of a subdivision scheme/cascade algorithm in $L_{p}$ :

the refinable function $\varphi$ belongs to $L_{p}(C$ for $p=\infty)$ and $\mathbf{m}(-1)=0$.

However, these conditions are not sufficient. The corresponding counterexamples are well known [3, 26]. The results of Chapter I make it possible to derive a sharp criterion of convergence and to compute the rate of convergence. We formulate all the results in the most general situation, i.e., for convergence in the spaces $C^{l}$ and $W_{p}^{l}$ for $l \geq 0$. As usual, convergence in $C^{l}$ means uniform convergence with the first $l$ derivatives, and that in $W_{p}^{l}$ means uniform convergence of the first $(l-1)$ derivatives and convergence of the $l$ th derivative in $L_{p}$. For functions on a segment, convergence in $C^{l}$ implies convergence in $W_{p}^{l}$, which, in its turn, implies convergence in $W_{1}^{l}$. For this reason, we shall formulate the necessary conditions for the weakest convergence (in $W_{1}^{l}$ ) and the sufficient conditions for the strongest convergence (in $C^{l}$ ). Throughout in the sequel, $l \geq 0$ is arbitrary; for $l=0$ we obtain results on convergence in $C$ and $L_{p}$. We start with the following fact proved in 3 .

Proposition 13. If for some $g \in W_{1}^{l}$ we have $T^{n} g \rightarrow 0$ in $W_{1}^{l}$, then the Fourier transform $\widehat{g}(\xi)$ vanishes at the point $\xi=0$ and has zeros of order at least $l+1$ at all points $k \in$ $\mathbb{Z} \backslash\{0\}$.

Given $l \geq 0$ and $p \in[0,+\infty]$, we let $\mathcal{L}_{p}^{l}$ denote the space of functions $g$ in $W_{p}^{l}$ (in $C^{l}$ for $p=\infty$ ) such that the Fourier transform of the derivative $g^{(l)}$ has zeros of order at least $l+1$ at all the points $k \in \mathbb{Z}$. By the Poisson summation formula, this condition is equivalent to

$$
\sum_{k \in \mathbb{Z}} k^{r} g^{(l)}(\cdot-k)=0, \quad r=0, \ldots, l .
$$

Thus, $\mathcal{L}_{p}^{l}$ is a subspace of codimension $l+1$ in the space of compactly supported functions in $W_{p}^{l}$. By Proposition 13, if $T^{n} g \rightarrow 0$ in the space $W_{p}^{l}$, then $g \in \mathcal{L}_{p}^{l}$.

Lemma 9. If for some compactly supported function $f \in W_{1}^{l}$ we have $T^{n} f \rightarrow \varphi$ in $W_{1}^{l}$, then $g=f-\varphi \in \mathcal{L}_{1}^{l}$.

Proof. Since $T^{n} \varphi=\varphi$ and $T$ is linear, we have $T^{n} f-\varphi=T^{n}(f-\varphi)$. It remains to apply Proposition 13 to the function $g=f-\varphi$.

This fact makes it possible to define the convergence of a cascade algorithm/subdivision scheme in $W_{p}^{l}$ as follows:

Definition 1. A cascade algorithm/subdivision scheme converges in $W_{p}^{l}$ if $T^{n} g \rightarrow 0$ for all $g \in \mathcal{L}_{p}^{l}$.

If a cascade algorithm converges in $W_{p}^{l}$, then $\varphi \in W_{p}^{l}\left(C^{l}\right.$ for $\left.p=\infty\right)[3]$.

Lemma 10. If a cascade algorithm/subdivision scheme converges in $W_{1}^{l}$, then the symbol $\mathbf{m}$ has a root of multiplicity at least $l+1$ at $z=-1$, i.e., it is factored as

$$
\mathbf{m}(z)=\left(\frac{1+z}{2}\right)^{l+1} \mathbf{m}_{l+1}(z) .
$$


Proof. See [3].

Thus, if a cascade algorithm converges in $W_{1}^{l}$, then its symbol possesses a trivial cycle of order at least $l+1$. This means, in particular, that the operator $T$ respects all the subspaces $\mathcal{L}_{p}^{l}$.

Let $\mathbf{m}_{l}(z)=\left(\frac{2}{z+1}\right)^{l} \mathbf{m}(z)$. The next statement is similar to Theorem 6 it reduces convergence in $W_{p}^{l}$ to that in $L_{p}$.

Lemma 11. The cascade algorithm with symbol $\mathbf{m}$ converges in $W_{p}^{l}$ if and only if the algorithm with symbol $\mathbf{m}_{l}$ converges in $L_{p}$.

Proof. The operator $\mathcal{S}$ of convolution with the function $\chi_{[0,1]}$ is a homeomorphism between the spaces $\mathcal{L}_{p}^{k}$ and $\mathcal{L}_{p}^{k+1}, k \geq 0$. Hence, $\mathcal{S}^{l}$ is a homeomorphism between $\mathcal{L}_{p}^{0}$ and $\mathcal{L}_{p}^{l}$. Let $T^{(l)}$ denote the transition operator corresponding to the symbol $\mathbf{m}_{l}$. The restriction of $T$ to $\mathcal{L}_{p}^{l}$ is isomorphic to the restriction of $T^{(l)}$ to $\mathcal{L}_{p}^{0}$. Furthermore,

$$
\begin{aligned}
& T^{(l)} f=\mathcal{S}^{-l} T \mathcal{S}^{l} f, \quad f \in \mathcal{L}_{p}^{0}, \\
& T g=\mathcal{S}^{l} T^{(l)} \mathcal{S}^{-l} g, \quad g \in \mathcal{L}_{p}^{l} .
\end{aligned}
$$

To prove this, it suffices to establish the identity

$$
B_{l-1} * T^{(l)}[f]=T\left[B_{l-1} * f\right],
$$

by taking the Fourier transform of both sides $\left(B_{k}=\mathcal{S}^{k} \chi_{[0,1)}\right.$ is the cardinal $B$-spline).

Remark 5. Relation (48) shows that the convergence of the cascade algorithm in $W_{p}^{l}$ is equivalent to the fact that

$$
\left\|T^{n} B_{l}-\varphi\right\|_{W_{p}^{l}} \rightarrow 0, \quad n \rightarrow \infty
$$

where $B_{l}(x)$ is the cardinal $B$-spline. Thus, a cascade algorithm converges in $W_{p}^{l}$ if and only if the sequence $T^{n} B_{l}$ converges to $\varphi$ in that space. Actually, this is an equivalent definition of the convergence of cascade algorithms in $W_{p}^{l}$.

For simplicity, in the sequel we set $\mathcal{L}_{p}^{0}=\mathcal{L}_{p}$. Thus, the space $\mathcal{L}_{p}$ consists of compactly supported functions $g \in L_{p}$ for which $\sum_{k \in \mathbb{Z}} g(x-k)=0$ a.e. We denote by $\mathcal{L}_{p}[0, N]$ the space of functions in $\mathcal{L}_{p}$ supported on the segment $[0, N]$. The following result was proved in [2]. For given operators $A_{0}$ and $A_{1}$, we set

$$
\left\|\Sigma_{k}\left(A_{0}, A_{1}\right)\right\|_{p}=\left(2^{-k} \sum_{d_{1}, \ldots, d_{k}}\left\|A_{d_{1}} \cdots A_{d_{k}}\right\|^{p}\right)^{1 / p}
$$

(with the usual modifications for $p=\infty$ ). Let

$$
W=\left\{x \in \mathbb{R}^{N}, \sum x_{k}=0\right\} \quad \text { and } \quad\left\|\Sigma_{n}\right\|_{p}=\left\|\left(\left.T_{0}\right|_{W},\left.T_{1}\right|_{W}\right)\right\|_{p} .
$$

Proposition 14. A cascade algorithm converges in $L_{p}, p \in[1,+\infty]$, if and only if $\rho_{p}\left(\left.T_{0}\right|_{W},\left.T_{1}\right|_{W}\right)<1$. Moreover,

$$
\sup _{g \in \mathcal{L}_{p}[0, N],\|g\|_{p}=1}\left\|T^{n} g\right\|_{p} \asymp\left\|\Sigma_{n}\right\|_{p} .
$$

Remark 6. Proposition 14 explains why the condition $\varphi \in L_{p}$ is not sufficient for convergence in $L_{p}$. The function $\varphi$ belongs to $L_{p}$ provided the $p$-radius of the operators $T_{0}, T_{1}$ on the space $V$ is smaller than 1 (see Theorem 9). On the other hand, the convergence of the algorithm in $L_{p}$ means that the $p$-radius on the space $W$ is smaller than 1 . Since $V \subset W$, we see that the convergence implies that $\varphi \in L_{p}$, but not conversely. Theorem 7 
and Proposition 11 show that $V=W$ if and only if the symbol $\mathbf{m}$ has neither symmetric zeros nor nontrivial cycles. In this case convergence is indeed equivalent to the smoothness of the solution; this result originates in [3] for $\mathbf{p}=\infty$ and in [27. for finite $p$. In the general case, when $\mathbf{m}$ has nontrivial cycles and symmetric zeros, the embedding $V \subset W$ is strict, so that the $p$-radii on these subspaces may fail to coincide. The explicit form of $V$ obtained in $\S 8$ enables us to find the relationship between the two radii, and therefore, between the smoothness of refinable functions and the convergence of cascade algorithms.

Theorem 11. A cascade algorithm/subdivision scheme converges in $W_{p}^{l}$ if and only if the following three conditions are satisfied: $\varphi \in W_{p}^{l}\left(C^{l}\right.$ for $\left.p=\infty\right)$; the symbol $\mathbf{m}$ is factored as in (46); if $\mathbf{m}$ has nontrivial generalized cycles, then $\kappa_{\mathbf{b}}(\mathbf{m})<2^{-l}$ for each of them.

Proof. By Lemma 11 the proof is reduced to the case where $l=0$. By Proposition 14, the convergence of the algorithm in $L_{p}$ is equivalent to the inequality $\rho_{p}\left(\left.T_{0}\right|_{W},\left.T_{1}\right|_{W}\right)<1$. By Theorem 5 , the matrices of the operators $T_{0}, T_{1}$ in a special basis have the block lower triangular form (21). Consequently, the restrictions of these operators to the space $W$ are of the same form with the only difference being that the multiplicity of the trivial cycle $\mathbf{b}_{0}$ becomes reduced by 1 . Applying Lemma 8 , we see that $\rho_{p}\left(\left.T_{0}\right|_{W},\left.T_{1}\right|_{W}\right)$ is equal to the largest $p$-radius of the blocks in the factorization (21). For the blocks, we have $\rho_{p}\left(A_{j, 0}, A_{j, 1}\right)=\kappa_{\mathbf{b}_{j}}\left(\mathbf{m}_{1}\right)$ by (39). For the nontrivial cycles, we have $\kappa_{\mathbf{b}_{j}}\left(\mathbf{m}_{1}\right)=\kappa_{\mathbf{b}_{j}}(\mathbf{m})$, and for the trivial cycle we have $\kappa_{\mathbf{b}_{0}}\left(\mathbf{m}_{1}\right)=\frac{1}{2} \kappa_{\mathbf{b}_{0}}(\mathbf{m})=\frac{1}{2} \mathbf{m}(1)=\frac{1}{2}$ (see Proposition 6). Therefore,

$$
\rho_{p}\left(\left.T_{0}\right|_{W},\left.T_{1}\right|_{W}\right)=\max \left\{\rho_{p}\left(\tilde{T}_{0}, \tilde{T}_{1}\right), \frac{1}{2}, \kappa_{\mathbf{b}_{1}}(\mathbf{m}), \ldots, \kappa_{\mathbf{b}_{s}}(\mathbf{m})\right\},
$$

where $\mathbf{b}_{1}, \ldots, \mathbf{b}_{s}$ are the nontrivial generalized cycles of $\mathbf{m}$. Theorem 9 shows that $\rho_{p}\left(\tilde{T}_{0}, \tilde{T}_{1}\right)<1$, because $\varphi \in L_{p}$. Therefore, $\rho_{p}\left(\left.T_{0}\right|_{W},\left.T_{1}\right|_{W}\right)$ is smaller than 1 if and only if $\kappa_{\mathbf{b}_{j}}(\mathbf{m})<1$ for all $j$. The theorem follows.

The convergence problem for cascade algorithms was studied by many authors, e.g., by Daubechies and Lagarias [4, Cavaretta, Dahmen, and Micchelli [3, Jia [27, Wu 28, Chen and Hun [29, etc. In [26, Neamtu presented an incorrect solution of this problem.

Corollary 7. If the symbol $\mathbf{m}$ has no nontrivial generalized cycles, then the cascade algorithm converges in $W_{p}^{l}, l \geq 0$, if and only if $\varphi \in W_{p}^{l}\left(\varphi \in C^{l}\right.$ for $\left.p=\infty\right)$, and the symbol $\mathbf{m}$ can be factored as in (46).

For $l=0$, Corollary 7 was first established for stable refinable functions in [3]. It was shown that if $\mathbf{m}(-1)=0$ and the refinable function $\varphi$ is stable, then the convergence of the subdivision scheme in $C$ is equivalent to the continuity of $\varphi$. In 27 this result was extended to convergence in the spaces $L_{p}$. Corollary 7 generalizes this result to the spaces of higher smoothness for all $l \geq 1$ and, moreover, relaxes the assumptions on the symbol: now it suffices that the symbol has no generalized cycles, but it may have symmetric zeros.

Corollary 8. The symmetric zeros of the symbol do not influence the convergence of the cascade algorithm.

Remark 7. The assumptions of Theorem 11 can be checked easily for any symbol $\mathbf{m}(z)$. Since the total number of roots of $\mathbf{m}$ does not exceed its degree $N$, we see that it suffices to look over all cut sets $\mathcal{A}$ of the cyclic trees such that $\operatorname{Card} \mathcal{A} \leq N$, and to verify the condition $\mathbf{m}(\mathcal{A})=0$ for each of them. This condition can be written as a linear system 
for the coefficients $\left\{c_{k}\right\}$ of $\mathbf{m}(z)$. In this way, all the generalized cycles of the symbol $\mathbf{m}$ will be found. It remains to compute the values $\kappa_{\mathbf{b}}(\mathbf{m})$ and to compare them with $2^{-l}$.

\section{$\S 11$. EXAMPLES AND SPECIAL CASES}

In Examples 3 and 4 we apply the criterion of Theorem 11 to specific algorithms. Then we treat several special cases important in applications.

Example 3. The refinement equation

$$
\varphi(x)=\frac{1}{2}(\varphi(2 x)+\varphi(2 x-1)+\varphi(2 x-3)+\varphi(2 x-4))
$$

has a continuous solution $\varphi=\frac{1}{3} \chi_{[0,1]} * \chi_{[0,3]}$. This is a piecewise linear function. Thus, $\varphi \in C$ and $\varphi \in W_{p}^{1}$ for all $p$, although $\varphi \notin C^{1}$. The $\operatorname{symbol} \mathbf{m}(z)=\frac{1}{2}(z+1)\left(z^{3}+1\right)$ has a unique nontrivial generalized cycle $\mathbf{b}=\left\{e^{-\frac{2 \pi i}{3}}, e^{-\frac{4 \pi i}{3}}\right\}$, which is actually a usual cycle. A direct calculation yields $\kappa_{\mathbf{b}}(\mathbf{m})=\frac{1}{2}$. Hence, we have $\kappa_{\mathbf{b}}(\mathbf{m})<1$. Thus, the cascade algorithm converges in $C$, and therefore in $L_{p}$ for all $p$. We see that the generalized cycle has not influenced convergence in these spaces. However, it has "spoiled" convergence in $W_{p}^{1}$ for all $p \in[1,+\infty)$. Indeed, since $\kappa_{\mathbf{b}}(\mathbf{m})=2^{-1}$, the algorithm diverges in $W_{p}^{1}$, although $\varphi \in W_{p}^{1}$.

Multiplying the symbol by the polynomial $z^{2}-z+1=\left(z-e^{\frac{\pi i}{3}}\right)\left(z-e^{-\frac{\pi i}{3}}\right)$, we arrive at the new symbol $\mathbf{m}_{1}(z)=\frac{1}{2}\left(z^{3}+1\right)^{2}$. The corresponding equation also possesses a piecewise linear solution $\varphi_{1}(x)=\frac{1}{9} \chi_{[0,3]} * \chi_{[0,3]}=\frac{1}{3} B_{1}\left(\frac{x}{3}\right)$. However, the cascade algorithm converges neither in $C$ nor in $L_{1}$. Indeed, $\mathbf{m}_{1}$ has the same generalized cycle $\mathbf{b}$, but of multiplicity 2 , and now we have $\kappa_{\mathbf{b}}\left(\mathbf{m}_{1}\right)=1$.

Example 4. The equation corresponding to the symbol

$$
\mathbf{m}(z)=\left(\frac{2}{5}\left(z^{5}+1\right)+\frac{1}{20}\left(z+z^{2}+z^{3}+z^{4}\right)\right)\left(z^{8}-z^{6}+z^{4}-z^{2}+1\right)
$$

has a continuous solution, but the cascade algorithm diverges in $C$. Denote the first factor by $\mathbf{m}_{1}(z)$ and the second by $\mathbf{p}_{1}(z)$. The polynomial $\mathbf{m}_{1}(z)$ has positive coefficients and $\mathbf{m}_{1}(-1)=0$. Therefore, the solution $\varphi_{1}$ of the corresponding equation is continuous (a refinable function corresponding to a positive mask satisfying (42) is continuous; see [18]). The second factor $\mathbf{p}_{1}(z)$ has 8 roots $e^{\frac{(2 k+1) \pi i}{10}}, k \in\{0, \ldots, 9\}, k \neq 2,7$. They form a cut set $\mathcal{A}$ for the generalized cycle $\mathbf{b}=\left\{e^{-\frac{2 \pi i}{5}}, e^{-\frac{4 \pi i}{5}}, e^{-\frac{8 \pi i}{5}}, e^{-\frac{16 \pi i}{5}}\right\}$. Thus, $\mathbf{m}$ possesses a generalized cycle $\mathbf{b}$ of multiplicity 1 . Removing this cycle, we obtain the equation with the symbol $\mathbf{m}_{1}$ and with the continuous solution $\varphi_{1}$. Therefore, the solution $\varphi$ of the initial equation is continuous as well. It is easy to check that $\kappa_{\mathbf{b}}(\mathbf{m})>1.2$. Hence, the cascade algorithm diverges.

Interpolatory subdivision schemes. A mask is said to be interpolational if $c_{k}=1$ for some $k$ and $c_{k+2 j}=0$ for all $j$. Since the symbol of such a mask has no symmetric zeros, it follows that its generalized cycles are usual ones. If its symbol has no cycles, then the cascade algorithm converges provided the refinable function belongs to the corresponding space. If the symbol has a cycle $\mathbf{b}$, then for any element $b \in \mathbf{b}$ we have $\mathbf{m}(b)=b^{k}+\mathbf{m}(-b)=b^{k}$ (because $\left.\mathbf{m}(z)-\mathbf{m}(-z) \equiv z^{k}\right)$. Consequently, $|\mathbf{m}(b)|=1$. Thus, we have $\kappa_{\mathbf{b}}(\mathbf{m})=1$, and Theorem 11 shows that the algorithm diverges.

Corollary 9. An interpolation scheme converges in $C$ (or in $L_{p}$ ) if and only if the corresponding refinable function belongs to $C$ (respectively, $L_{p}$ ) and the symbol has no nontrivial cycles. 
Sparse equations. Sparse symbols are of the form $\mathbf{m}(z)=\mathbf{m}_{1}\left(z^{k}\right)$, where $k \geq 2$ is an integer, and $\mathbf{m}_{1}$ is a symbol satisfying the usual assumptions $\mathbf{m}_{1}(1)=1, \mathbf{m}_{1}(-1)=0$. Observe that the equations with symbols $\mathbf{m}$ and $\mathbf{m}_{1}$ have solutions of equal regularity, because one of them is obtained from the other by the $k$ times dilation of the argument. Therefore, spare equations may have smooth solutions. However, their cascade algorithms always diverge even in $L_{1}$. In the case of even $k$ the necessary condition $\mathbf{m}(-1)=0$ fails, because $\mathbf{m}(-1)=\mathbf{m}_{1}(1)=1$. If $k$ is odd, then the set $e^{-2 \pi r i / k}, r=0, \ldots, k-1$ (the vertices of a regular $k$-gon on the unit circle) are naturally split into several cyclic sets. Each of them is a usual cycle of $\mathbf{m}$. Indeed, $\mathbf{m}\left(-e^{-2 \pi r i / k}\right)=\mathbf{m}_{1}\left(-e^{-2 \pi r i}\right)=\mathbf{m}_{1}(-1)=$ 0 . On the other hand, $\mathbf{m}\left(e^{-2 \pi r i / k}\right)=\mathbf{m}_{1}\left(e^{-2 \pi r i}\right)=\mathbf{m}_{1}(1)=1$, so that for any of these cycles we have $\kappa_{\mathbf{b}}(\mathbf{m})=1$. Thus, by Theorem 11, the cascade algorithm diverges.

Equations with nonnegative masks. If all the coefficients $c_{k}$ are nonnegative, then, in the case of finite $p$, the convergence of the subdivision scheme in $L_{p}$ can be ensured without computing the $p$-radius.

Theorem 12. Suppose all the coefficients of a refinement equation are nonnegative; then the cascade algorithm converges in $L_{p}, p \in[1,+\infty)$, if and only if the equation is not sparse and $\mathbf{m}(-1)=0$.

Proof. The "only if" part is obvious; we establish the "if" part. First, observe that if the coefficients are nonnegative and $\mathbf{m}(-1)=0$, then the solution belongs to $L_{\infty}$, and therefore, to all $L_{p}$ [11. Denote by $I$ the set of all indices $k$ for which $c_{k} \neq 0$. In particular, $0 \in I$. If the algorithm diverges, then, by Theorem 11, the symbol $\mathbf{m}$ must have a generalized cycle $\mathbf{b}$ such that $\kappa_{\mathbf{b}}(\mathbf{m}) \geq 1$. This is impossible, because $|\mathbf{m}(z)|<1$ for all points $z$ of the unit circle except for $z=1$. Indeed, since all the coefficients of $\mathbf{m}$ are nonnegative, for any $z$ with $|z|=1$ we have

$$
|\mathbf{m}(z)|=\left|\sum_{k} \frac{1}{2} c_{k} z^{k}\right| \leq \frac{1}{2} \sum_{k} c_{k}=1 .
$$

Since $c_{0}>0$, it follows that equality occurs only if $z^{k}=1$ for all $k \in I$. The greatest common divisor of the elements of $I$ is 1 (otherwise the mask is sparse). Therefore, $z=1$.

A divergent algorithm with a simple eigenvalue. The existence of divergent subdivision schemes with smooth refinable functions was observed in [3, 4]. All the corresponding examples in the literature originated from one special case when one of the operators $T_{i}, i=0,1$, has a multiple eigenvalue 1 . In this case $\rho\left(\left.T_{i}\right|_{W}\right) \geq 1$ and the algorithm diverges in $C$ by Proposition 14, In particular, the interpolation and sparse masks (see Examples 3 4 and Corollary 13) also correspond to this case. In [26], Neamtu posed a natural problem: does this case exhaust all possible divergent algorithms with smooth refinable functions? The example below shows that the answer is in the negative.

Example 5. The symbol $\mathbf{m}(z)=\left(0.3 z^{2}+0.5 z+0.2\right)\left(z-e^{-\frac{\pi i}{3}}\right)^{2}\left(z^{2}-e^{\frac{\pi i}{3}}\right)^{2}$ has the only nontrivial cycle of multiplicity 2 , namely, $\mathbf{b}=\left\{e^{\frac{2 \pi i}{3}}, e^{\frac{4 \pi i}{3}}\right\}$, with the cut set $\mathcal{A}=$ $\left\{e^{\frac{\pi i}{6}}, e^{\frac{7 \pi i}{6}}, e^{-\frac{\pi i}{3}}\right\}$. Removing this cycle, we obtain the symbol $\mathbf{m}_{\mathbf{b}}(z)=0.3 z^{2}+0.5 z+0.2$. Since its coefficients are positive, it follows that the solution $\varphi_{\mathbf{b}}$ is continuous [18. Hence, the solution of the initial equation $\varphi$ is also continuous. Since $\kappa_{\mathbf{b}}(\mathbf{m})=\sqrt{1.12}>1$, we see that the cascade algorithm diverges even in $L_{1}$. On the other hand, by using the factorization (21), it is easy to show that the modules of the eigenvalues of $T_{0}$ are equal to $1,0.4, \sqrt{1.12}, \frac{1}{2} \sqrt{1.12}, 0$, and 0 , respectively, while the modules of the eigenvalues of $T_{1}$ are $1,0.6, \sqrt{1.12}, \frac{1}{2} \sqrt{1.12}, 0$, and 0 . Thus, 1 is a simple eigenvalue for both $T_{0}$ and $T_{1}$. 


\section{§12. The Rate of COnVERgence}

Definition 2. The rate of convergence of a cascade algorithm/subdividion scheme in the space $L_{p}, p \in[1,+\infty]$, is the quantity

$$
\nu_{p}=l+\inf _{g \in \mathcal{L}_{p}^{l}[0, N]} \lim _{k \rightarrow \infty}-k^{-1} \log _{2}\left\|T^{k} g\right\|_{W_{p}^{l}},
$$

where $l \geq 0$ is the largest integer for which the algorithm converges in $W_{p}^{l}$.

Proposition 14 yields the following.

Corollary 10. Suppose $l \geq 0$ and $p \in[1,+\infty]$. A cascade algorithm converges in $W_{p}^{l}$ if and only if the corresponding symbol $\mathbf{m}$ can be factored as in (46) and $\rho_{p}=$ $\rho_{p}\left(T_{0}^{(l+1)}, T_{1}^{(l+1)}\right)<1$, where $T_{0}^{(l+1)}, T_{1}^{(l+1)}$ are the matrices of the symbol $\mathbf{m}_{l+1}$. Moreover, $\nu_{p}=l-\log _{2} \rho_{p}$, where $l$ is the largest integer for which the algorithm converges in $W_{p}^{l}$.

Corollary 11. A cascade algorithm converges in $W_{p}^{l}$ if and only if $\nu_{p}>l$.

For example, if $\nu_{\infty}=3$, then the algorithm converges in $C^{2}(\mathbb{R})$, but not in $C^{3}(\mathbb{R})$.

Example 6. Consider the subdivision scheme for the symbol $\mathbf{m}(z)=0.4 z^{2}+0.5 z+0.1$. Since the multiplicity of the root $z=-1$ is one, the scheme diverges in $W_{p}^{1}$, and $\nu_{p} \leq 1$ for all $p$. From the factorization $\mathbf{m}(z)=\left(\frac{z+1}{2}\right)(0.8 z+0.2)$ it follows that $T_{0}^{(1)}=0.2$ and $T_{1}^{(1)}=0.8$ (both operators are one-dimensional). We have $\rho_{p}=\left(\frac{0.8^{p}+0.2^{p}}{2}\right)^{1 / p}$ and $\rho_{\infty}=0.8$. Thus, $\nu_{\infty}=-\log _{2} 0.8=0.3219 \ldots$ For $p=2$ we have $\nu_{2}=-\log _{2} \sqrt{0.34}=$ $0.778 \ldots$, and for $p=1$ we have $\nu_{1}=1$.

Now we are able to express the rate of convergence of the cascade algorithm in terms of the regularity of the refinable function. Let $\mathbf{l}(\mathbf{m})$ denote the number that is smaller by one than the multiplicity of the root $z=-1$. Thus, $\mathbf{l}(\mathbf{m})$ is the largest possible order $l$ in the factorization (46).

Theorem 13. If a refinable function $\varphi$ belongs to $L_{p}(C$ for $p=+\infty)$, then

$$
\nu_{p}=\min \left\{\alpha_{\varphi, p}, \mathbf{l}(\mathbf{m})+1,-\log _{2} \kappa_{\mathbf{b}_{1}}(\mathbf{m}), \ldots,-\log _{2} \kappa_{\mathbf{b}_{s}}(\mathbf{m})\right\},
$$

where $\mathbf{b}_{1}, \ldots, \mathbf{b}_{s}$ are the nontrivial generalized cycles of the symbol $\mathbf{m}$.

Proof. We denote by $M$ the value of the right-hand side in (53) and by $l$ the largest integer not exceeding $M$. By Theorem [1] if $M \leq 0$, then the algorithm diverges, and there is nothing to prove. Assume $M>0$. By Theorem [11, the algorithm converges in $W_{p}^{l}$ and diverges in $W_{p}^{l+1}$. By Lemma 11, it suffices to consider the case of $l=0$. We have $\mathbf{l}(\mathbf{m})+1 \geq 1$. Proposition 14 yields

$$
\nu_{p}=-\log _{2} \rho_{p}\left(\left.T_{0}\right|_{W},\left.T_{1}\right|_{W}\right) .
$$

Invoking (51) and observing that $\alpha_{\varphi, p}=-\log _{2} \rho_{p}\left(\tilde{T}_{0}, \tilde{T}_{1}\right)$ (see Theorem 9), we conclude the proof.

Corollary 12. If a symbol has no nontrivial generalized cycles, then

$$
\nu_{p}=\min \left\{\alpha_{\varphi, p}, \mathbf{l}(\mathbf{m})+1\right\}, \quad p \in[1,+\infty] .
$$

Thus, the quantity $\nu_{p}$ characterizes the exponential rate of the convergence $T^{n} B_{l} \rightarrow \varphi$ in the metric $W_{p}^{l}$ as $n \rightarrow \infty$. This convergence occurs in $L_{p}$ (in $W_{p}^{l}$ ) if and only if $\nu_{p}>0$ (respectively, $\nu_{p}>l$ ). A sharper estimate is given in the following statement. 
Proposition 15. Let $p \in[1,+\infty]$, and let $l \geq 0$ be the largest integer for which the cascade algorithm converges in $W_{p}^{l}$. Then

$$
c_{1} \cdot 2^{\left(l-\nu_{p}\right) n} \leq\left\|T^{n} B_{l}-\varphi\right\|_{W_{p}^{l}} \leq c_{2} \cdot n^{s-1} \cdot 2^{\left(l-\nu_{p}\right) n}
$$

for any $n$, where $c_{1}, c_{2}$ are positive constants, and $s$ is the number of elements of the set $\alpha_{\varphi, p}, \mathbf{l}(\mathbf{m})+1,-\log _{2} \kappa_{\mathbf{b}_{1}}(\mathbf{m}), \ldots,-\log _{2} \kappa_{\mathbf{b}_{s}}(\mathbf{m})$ that realize the minimum on the right-hand side of (53).

Proof. Using Lemma 11 once again, we restrict ourselves to the case where $l=0$. Combining (50) and Proposition 12, we see that the quantity $\left\|T^{n} B_{l}-\varphi\right\|_{L_{p}}$ lies between $C_{1} \rho_{p}^{n}$ and $C_{2} n^{s-1} \rho_{p}^{n}$, where $\rho_{p}$ is the $p$-radius of the operators $\left.T_{i}\right|_{W}, i=0,1$, and $s$ is their valency. The factorization (21) shows that the $p$-radii of the blocks are $2^{-\alpha_{\varphi}}, 2^{-\mathbf{l}(\mathbf{m})-1}$, $\kappa_{\mathbf{b}_{1}}(\mathbf{m}), \ldots, \kappa_{\mathbf{b}_{s}}\left(\mathbf{m}_{1}\right)$, respectively, and the proposition follows.

\section{§13. SharpNess OF THE CONVERGENCE CRITERION}

Isn't the criterion of Theorem 11 too complicated for characterizing the divergent cascade algorithms? Do we really need all possible generalized cycles for that? In fact, the divergence implies that the symbol has a generalized cycle $\mathbf{b}$ such that $\kappa_{\mathbf{b}}(\mathbf{m}) \geq 1$. However, is this property compatible with the smoothness of the refinable function $\varphi$ ?

It turns out that every generalized cycle can cause the divergence of some cascade algorithm with a smooth refinable function. In this sense, the criterion of Theorem 11 is complete and sharp. For simplicity, we formulate the result for the case where $l=0$, and $p=\infty$, i.e., for convergence in $C$.

Proposition 16. Let $\mathbf{b}$ be an arbitrary cyclic set different from $\{1\}$, and let $\mathcal{A}$ be an arbitrary cut set of the corresponding tree $\mathcal{T}_{\mathbf{b}}$. Then there exists a refinement equation with symbol $\mathbf{m}(z)$ such that

1) $\mathbf{m}(\mathcal{A})=0$, i.e., $\mathbf{b}$ is a generalized cycle of $\mathbf{m}$ with cut set $\mathcal{A}$;

2 ) the refinable function is continuous, but the corresponding algorithm diverges in $C$;

3) after removing this generalized cycle, the algorithm becomes convergent.

Proof. Let $\mathbf{p}(z)=\left(\frac{1+z}{2}\right) \mathbf{a}(z)$ be a polynomial such that $\mathbf{a}(1)=1, \operatorname{deg} \mathbf{a} \geq 2$, and the cascade algorithm with symbol $\mathbf{p}$ converges in $C$. For instance, any polynomial a with positive coefficients fits. Then after any small perturbation of the coefficients of a the algorithm $\mathbf{p}$ still converges in $C$. Indeed, convergence is equivalent to the inequality $\rho_{\infty}\left(T_{0}^{(a)}, T_{1}^{(a)}\right)<1$, where $T_{i}^{(a)}$ is the matrix of the symbol a (see Proposition 14), and the quantity $\rho_{\infty}$ is continuous with respect to operators [5. With possible perturbation of the polynomial $\mathbf{a}(z)$, it may be assumed that $\mathbf{a}(z)$ has no roots on the unit circle and the number $\kappa_{\mathbf{b}}(\mathbf{a})$ is irrational. The last assumption can be ensured by the intermediate value theorem. In particular, $\kappa_{\mathbf{b}}(\mathbf{a})>0$, whence $\kappa_{\mathbf{b}}(\mathbf{m})>0$. Suppose $\mathbf{q}(z)=\prod_{\alpha \in \mathcal{A}}(z-\alpha)$; then $\kappa_{\mathbf{b}}\left(\mathbf{p q}^{r}\right)=2^{r} \kappa_{\mathbf{b}}(\mathbf{p})$ by Proposition 6. Let $r$ be the smallest positive integer such that $\kappa_{\mathbf{b}}\left(\mathbf{p q}^{r}\right)>1$. Denote $\mathbf{a}_{r-1}=\mathbf{a q}^{r-1}$ and $\mathbf{p}_{r-1}=\mathbf{p q}^{r-1}$. Since the number $\kappa_{\mathbf{b}}\left(\mathbf{p}_{r-1}\right)$ is irrational, it is not equal to 1 . Therefore, $\kappa_{\mathbf{b}}\left(\mathbf{p}_{r-1}\right)<1$. However, since $\mathbf{b}$ is the only generalized cycle of the polynomial $\mathbf{p}_{r-1}$, it follows that the cascade algorithm with symbol $\mathbf{p}_{r-1}$ converges in $C$. Now we perturb the polynomial $\mathbf{a}_{r-1}$ slightly so that the algorithm with symbol $\mathbf{p}_{r-1}$ still converges, the number $\kappa_{\mathbf{b}}\left(\mathbf{p}_{r-1} \mathbf{q}\right)$ still exceeds 1 , but $\mathbf{a}_{r-1}$ does not vanish on the unit circle. Then the symbol $\mathbf{m}=\mathbf{p}_{r-1} \mathbf{q}$ has a unique generalized cycle $\mathbf{b}$ with the cut set $\mathcal{A}$. Since $\kappa_{\mathbf{b}}(\mathbf{m})>1$, the algorithm with symbol $\mathbf{m}$ diverges. Nevertheless, removing this generalized cycle, we get the symbol $\mathbf{p}_{r-1}$ with a converging cascade algorithm. 
Remark 8. Using the same method, we construct examples of symbols whose cascade algorithms diverge in $C$, but become convergent in $C^{l}$ after removing a given generalized cycle b. For this, we can take the polynomial $\mathbf{p}(\xi)=\left(\frac{1+z}{2}\right)^{l+1} \mathbf{a}(\xi)$, obtaining the corresponding polynomials $\mathbf{p}_{r-1}$ and $\mathbf{m}$ in the same way as above. However, in this case $\mathbf{b}$ must have multiplicity at least $l+1$. Why cannot we manage with cycles of multiplicity 1 ? The answer is given below.

Proposition 17. For a given $l \geq 0$, assume that a refinable function belongs to $C^{l}$ and the symbol satisfies (46). If the algorithm diverges in $C$, then the symbol has a generalized cycle of multiplicity at least $l+1$.

In the proof we use the following lemma established in 24 .

Lemma 12. If the refinement equation with symbol $\mathbf{m}$ has a solution $\varphi \in W_{1}^{l}$, then, for any cyclic set $\mathbf{b}$, either $\kappa_{\mathbf{b}}(\mathbf{m})<2^{-l}$, or $\mathbf{b}$ is a generalized cycle of $\mathbf{m}$ of multiplicity at least $l+1+\log _{2} \kappa_{\mathbf{b}}(\mathbf{m})$.

Proof of Proposition 17. If the cascade algorithm diverges in $C$, then, by Theorem 11. there is a generalized cycle $\mathbf{b}$ such that $\kappa_{\mathbf{b}}(\mathbf{m}) \geq 1$. Denote its multiplicity by $r$. We show that $r \geq l+1$. Deleting this generalized cycle (with its multiplicity), we obtain a symbol $\mathbf{m}_{\mathbf{b}}$. For this symbol, the refinable function also belongs to $C^{l}$ (see Proposition 10) and $\mathbf{b}$ is not a generalized cycle. By Lemma 12, we have $\kappa_{\mathbf{b}}\left(\mathbf{m}_{\mathbf{b}}\right)<2^{-l}$. Applying Proposition 6, we get $\kappa_{\mathbf{b}}(\mathbf{m})=2^{r} \kappa_{\mathbf{b}}\left(\mathbf{m}_{\mathbf{b}}\right)<2^{r-l}$. If $r \leq l$, then $\kappa_{\mathbf{b}}(\mathbf{m})<1$, which contradicts the choice of $\mathbf{b}$. Thus, $r \geq l+1$.

\section{Chapter IV. Properties and structure of Refinable functions}

\section{$\S 14$. Dependence of SOLUTIONS On COEFFICIENTS OF THE EQUATION}

In the space of tempered distributions $\mathcal{S}^{\prime}(\mathbb{R})$, the solution of a refinement equation is continuous with respect to the coefficients $\left\{c_{k}\right\}$. If a sequence of polynomials $\left\{\mathbf{m}_{j}(z)\right\}_{j \in \mathbb{N}}$ converges to the polynomial $\mathbf{m}(z)$ and for all $j$ we have $\operatorname{deg} \mathbf{m}_{j}=N$ and $\mathbf{m}_{j}(1)=1$, then the refinable functions $\varphi_{j}$ corresponding to these symbols converge to $\varphi$ in $\mathcal{S}^{\prime}$ (see [25, Subsection 2.4]). However, in other function spaces, for example, in $C$ or in $L_{p}$, solutions of refinement equations may fail to be continuous with respect to the coefficients. For instance, Lemma 7 says that there are arbitrarily small perturbations of coefficients making the solution purely singular. A natural question arises: what perturbations change the solutions continuously?

We are interested in continuity in the spaces $W_{p}^{l}$ for $l \geq 0$ and $p \in[1,+\infty]$; as usual, we replace $W_{\infty}^{l}$ by $C^{l}$. It turns out that for all $p, l$, the answer depends entirely on which roots of the symbol $\mathbf{m}$ are fixed and which roots are moved by the perturbation in question. For simplicity, we formulate the results in the space $C$, i.e., for the case where $l=0$ and $p=+\infty$. Then, we shall point out how to extend those results to the spaces $W_{p}^{l}$.

Suppose a small perturbation of $\mathbf{m}$ leads to a symbol $\mathbf{m}_{\varepsilon}$ :

$$
\left\|\mathbf{m}-\mathbf{m}_{\varepsilon}\right\|_{\infty}<\varepsilon, \quad \operatorname{deg} \mathbf{m}_{\varepsilon}=\operatorname{deg} \mathbf{m}, \quad \mathbf{m}_{\varepsilon}(1)=\mathbf{m}(1)=1,
$$

where $\|\mathbf{p}\|_{\infty}=\sup _{\xi \in \mathbb{R}}\left|\mathbf{p}\left(e^{2 \pi i \xi}\right)\right|$ is the uniform norm on the unit circle. We denote by $\varphi_{\varepsilon}$ the solution of the refinement equation with the symbol $\mathbf{m}_{\varepsilon}$. Under what conditions on $\mathbf{m}_{\varepsilon}$ does the convergence $\left\|\mathbf{m}-\mathbf{m}_{\varepsilon}\right\|_{\infty} \rightarrow 0$ as $\varepsilon \rightarrow 0$ imply that $\left\|\varphi-\varphi_{\varepsilon}\right\|_{\infty} \rightarrow 0$ ?

Let $\mathbf{b}_{0}, \ldots, \mathbf{b}_{s}$ be the generalized cycles of $\mathbf{m}$, and let $r_{0}, \ldots, r_{s}$ be their multiplicities. The generalized cycle $\mathbf{b}_{0}$ is trivial; the others are nontrivial and distinct. $\operatorname{By~} \operatorname{GCD}\left(\mathbf{m}, \mathbf{m}_{\varepsilon}\right)$ we denote the polynomial that is the greatest common divisor of $\mathbf{m}$ and $\mathbf{m}_{\varepsilon}$ and has the leading coefficient 1 . Let $r_{j}^{\prime}$ be the multiplicity of the generalized cycle $\mathbf{b}_{j}$ of the 
polynomial $\operatorname{GCD}\left(\mathbf{m}, \mathbf{m}_{\varepsilon}\right)$ for $j=0, \ldots, s$. If $\mathbf{b}_{j}$ is not a generalized cycle, then we set $r_{j}^{\prime}=0$. Clearly, $r_{j}^{\prime} \leq r_{j}$ for all $j$. Thus, the numbers $r_{j}^{\prime}$ indicate which generalized cycles have survived after the transfer from $\mathbf{m}$ to $\mathbf{m}_{\varepsilon}$ and how their multiplicities have been reduced. These numbers depend only on the remaining generalized cycles of $\mathbf{m}$.

Theorem 14. Let $\varphi \in C(\mathbb{R})$ be a solution of a refinement equation. This solution varies continuously in $C(\mathbb{R})$ under a small perturbation of the coefficients if the perturbation is such that $r_{0}^{\prime} \geq 1$ and $r_{j}^{\prime}>-\log _{2} \kappa_{\mathbf{b}_{j}}(\mathbf{m})$ for all $j=1, \ldots, s$.

Conversely, there exists $\varepsilon>0$ such that if (56) is fulfilled and $\varphi_{\varepsilon} \in C(\mathbb{R})$, then $r_{0}^{\prime} \geq 1$ and $r_{j}^{\prime} \geq-\log _{2} \kappa_{\mathbf{b}}(\mathbf{m})$ for all $j=1, \ldots, s$.

Proof. Sufficiency. Assume the conditions $r_{0}^{\prime} \geq 1$ and $r_{j}^{\prime}>-\log _{2} \kappa_{\mathbf{b}}(\mathbf{m})$ are satisfied for all $j=1, \ldots, s$. We show that such perturbations change the function $\varphi$ continuously. First, we suppose that the corresponding cascade algorithm converges. Since the rate of convergence is $\nu=\log _{2} \rho_{\infty}$, where $\rho_{\infty}=\rho_{\infty}\left(\left.T_{0}\right|_{W},\left.T_{0}\right|_{W}\right)<1$, it follows that for any $q \in\left(\rho_{\infty}, 1\right)$ we have $\left\|T^{k} B_{0}-\varphi\right\|_{\infty}<C q^{k}$, where $B_{0}(x)=\chi_{[0,1)}$. Then

$$
\varphi=B_{0}+\sum_{j=0}^{\infty} T^{j}\left(T B_{0}-B_{0}\right) .
$$

If we perturb the coefficients of the symbol $\mathbf{m}$ so that at least one trivial cycle survives $\left(r_{0}^{\prime} \geq 1\right)$, then the operators $T_{i}$ will still have a common invariant subspace $W$. Moreover, the joint spectral radius on this subspace changes slightly, because $\rho_{\infty}$ is a continuous function of operators. Therefore, for all sufficiently small perturbations we have $\rho_{\infty}<q$. Taking $\delta>0$ arbitrarily, we choose $k$ such that $C \frac{q^{k+1}}{1-q}<\frac{\delta}{2}$. After a small perturbation, each of the first $k$ terms in (57) changes by at most $\frac{\delta}{2 k}$, and the sum of the norms of the other terms does not exceed $C q^{k+1} /(1-q)<\frac{\delta}{2}$. Thus, $\left\|\varphi-\varphi_{\varepsilon}\right\|<\delta$.

We pass to the general case. For every $j=1, \ldots, s$ we denote by $\mathcal{A}_{j}$ a cut set of multiplicity $r_{j}^{\prime}$ of the cyclic tree $\mathcal{T}_{\mathbf{b}_{j}}$; this cut set is assumed to consist of the roots of $\mathbf{m}$ that survived after the perturbation. In other words, $\mathcal{A}_{j}$ consists of the roots of the polynomial $\operatorname{GCD}\left(\mathbf{m}, \mathbf{m}_{\varepsilon}\right)$. Let $\mathcal{A}_{0}$ be the cut set of multiplicity 1 of $\mathcal{T}_{\mathbf{b}_{0}}=\mathcal{T}_{\{1\}}$ that consists of the roots of $\mathbf{m}$ that survived after the perturbation. Removing consecutively all symmetric zeros of $\mathbf{m}$ that lie in $\mathcal{A}_{j}$, we make all $\mathbf{b}_{j}$ usual cycles of multiplicities $1, r_{1}^{\prime}, \ldots, r_{s}^{\prime}$, respectively. Then we remove these cycles with their multiplicities. As a result, we obtain a symbol $\mathbf{m}_{u}(z)$. This symbol changes continuously under the admissible perturbations of the initial symbol $\mathbf{m}$. The assumptions imposed on the multiplicities $r_{j}^{\prime}$ imply that $\kappa_{\mathbf{b}_{j}}\left(\mathbf{m}_{u}\right)<1$ for all $j=1, \ldots, s$. By Theorem 11, the cascade algorithm with symbol $\mathbf{m}_{u}$ converges. Therefore, the corresponding refinable function $\varphi_{u}$ varies continuously under small perturbations of $\mathbf{m}_{u}$, and hence, under small perturbations of $\mathbf{m}$. The initial function $\varphi$ is obtained from $\varphi_{u}$ by formulas (24) and (25). Thus, $\varphi$ is a linear combination (with constant coefficients) of integral translates of the function $\varphi_{u}$; hence, it also changes continuously.

Necessity. Let $\Omega$ denote the union of all cut sets (of all possible cyclic trees) consisting of at most $N$ elements. Since the set $\Omega$ is finite, there exists a small $\varepsilon$ such that the symbol $\mathbf{m}_{\varepsilon}$ has no other roots belonging to $\Omega$ than those of $\mathbf{m}$ whenever $\left\|\mathbf{m}-\mathbf{m}_{\varepsilon}\right\|_{\infty}<\varepsilon$. Thus, all roots of $\mathbf{m}_{\varepsilon}$ in $\Omega$ are roots of $\mathbf{m}$. If $\varphi_{\varepsilon} \in C$, then $r_{0}^{\prime} \geq 1$ (see Lemma 7) and $\kappa_{\mathbf{b}_{j}}\left(\mathbf{m}_{\varepsilon}\right)<2^{-r_{j}^{\prime}}$ for all $j=1, \ldots, s$ (see Lemma 12). Since $\kappa_{\mathbf{b}_{j}}(\mathbf{m})$ is a continuous function of $\mathbf{m}$, we see that $2^{r_{j}^{\prime}} \geq \kappa_{\mathbf{b}_{j}}(\mathbf{m})$.

Remark 9. For all $p \in[1,+\infty)$, the conditions of continuity of refinable functions in $L_{p}$ with respect to the coefficients of the equations are the same as those in Theorem 14 for 
the space $C$. The conditions of continuity in the spaces $C^{l}$ and $W_{p}^{l}, p \in[1,+\infty)$, are as follows: $r_{0}^{\prime} \geq l+1$, and $r_{j}^{\prime}>l+\log _{2} \kappa_{\mathbf{b}_{j}}(\mathbf{m})$ for all $j=1, \ldots, s$ (in the necessary conditions the strict inequality is replaced by the nonstrict one). This is proved in the same way as in Theorem 14

Remark 10. Theorem 14 is "almost" a criterion for continuity of the solution with respect to the coefficients. It does not extend to the case where $r_{j}^{\prime}=\log _{2} \kappa_{\mathbf{b}_{j}}(\mathbf{m})$. In this case different situations are possible for the same equation: some perturbations of the coefficients change the function $\varphi$ continuously; others take $\varphi$ out of the space $C(\mathbb{R})$. Furthermore, there are perturbations that keep the function $\varphi$ in $C(\mathbb{R})$ but change it discontinuously.

Example 7. The symbol $\mathbf{m}(z)=\frac{1}{4} z^{6}+\frac{1}{2} z^{3}+\frac{1}{4}$ has cycles $\mathbf{b}_{0}=\{1\}, r_{0}=2$, and $\mathbf{b}_{1}=$ $\left\{e^{2 \pi i / 3}, e^{4 \pi i / 3}\right\}, r_{1}=2$. The solution $\varphi(\cdot)=B_{1}(\cdot / 3)$ is continuous. For any $\varepsilon \neq 0$ the perturbation

$$
\mathbf{m}_{\varepsilon}(z)=\mathbf{m}(z)+\varepsilon\left(z^{4}-z^{2}\right)
$$

takes the solution out of the space $C$. Indeed, since $\mathbf{b}_{1}$ is not a generalized cycle of $\mathbf{m}_{\varepsilon}$, and $\kappa_{\mathbf{b}_{1}}\left(\mathbf{m}_{\varepsilon}\right)=\sqrt{1+\varepsilon^{2}}>1$, we have $\varphi_{\varepsilon} \notin C(\mathbb{R})$ for any $\varepsilon \neq 0$ (see Lemma 12). On the other hand, for any $\varepsilon>0$ the perturbation

$$
\mathbf{m}_{\varepsilon}(z)=\left(\frac{1}{4}-\varepsilon\right) z^{6}+\frac{1}{2} z^{3}+\left(\frac{1}{4}-\varepsilon\right)+\varepsilon\left(z^{4}+z^{2}\right)
$$

keeps the solution $\varphi_{\varepsilon}$ in $C(\mathbb{R})$, but $\varphi_{\varepsilon}$ is discontinuous in $\varepsilon$ at the point $\varepsilon=0$. The function $\varphi_{\varepsilon}$ belongs to $C(\mathbb{R})$, because all the coefficients of the equation are nonnegative, and the indices of the nonzero coefficients are coprime. The continuity of solutions of such equations was proved by Melkman in [30] for $N \leq 8$ (in our case $N=6$ ). It is easy to check that for any $\varepsilon>0$ the matrix $T_{0}^{(\varepsilon)}$ has a unique (up to normalization) eigenvector with eigenvalue 1 . This is $v_{0}=(0,0,0,1,0,0)^{T}$. Therefore, for any $\varepsilon>0$ we have $\varphi_{\varepsilon}(k)=0$ for all integers $k \neq 0$. On the other hand,

$$
\varphi(k)=B_{1}(k / 3)= \begin{cases}k / 3 & \text { if } k=1,2,3 \\ (6-k) / 3 & \text { if } k=4,5,6\end{cases}
$$

Thus, the map $\varepsilon \rightarrow \varphi_{\varepsilon}$ from $\mathbb{R}_{+}$to $C(\mathbb{R})$ is discontinuous at the point $\varepsilon=0$.

Finally, the perturbation $\mathbf{m}_{\varepsilon}(z)=\left(\frac{1}{4}-\varepsilon\right) z^{6}+\varepsilon z^{5}+\varepsilon z^{4}+\left(\frac{1}{2}-2 \varepsilon\right) z^{3}+\varepsilon z^{2}+\varepsilon z+\left(\frac{1}{4}-\varepsilon\right)$ changes the solution continuously in $\varepsilon$ at the point $\varepsilon=0$. Observe that in all three of the cases above we have $r_{0}^{\prime}=1$ and $r_{1}^{\prime}=0$. Hence, the condition $r_{0}^{\prime} \geq 1$ is satisfied, and $2^{r_{1}^{\prime}}=\kappa_{\mathbf{b}_{1}}(\mathbf{m})=1$.

\section{§15. Construction of solutions}

A continuous solution $\varphi(x)$ of a refinement equation or, what is the same, the vectorfunction (30), is constructed pointwise. First, this function is computed at the dyadic points $x \in[0, N]$, and then it extends to the entire segment by continuity. The first step starts with finding the values at integral points: the vector $v_{0}=v(0)$ is an eigenvector of the matrix $T_{0}$ with eigenvalue 1 . After that, having found $v(0)$, we compute $v(x)$ at the diadic points $x=2^{-n} k=0 . d_{1} \cdots d_{n}$ by the formula

$$
v(x)=T_{d_{1}} \cdots T_{d_{n}} v_{0} \text {. }
$$

If the exact values of $v(x)$ for all $x=2^{-n} k$ are available, we get approximate values of the function on the entire interval with a prescribed accuracy, because the modulus of continuity of $\varphi$ is known from Theorem 10. Thus, if it is known that $\varphi$ is continuous, then its computation with a given error reduces to finding the vector $v_{0}$ and applying formula (58). The deviation from the true values on the entire segment tends to zero 
exponentially in $n$. Thus, the crucial point is to find the vector $v_{0}$. However, at this point we face unexpected difficulties. The reason is that the eigenvalue 1 may be multiple for the operator $T_{0}$. Accordingly, the eigenvectors corresponding to this eigenvalue may form a subspace of dimension greater than 1 .

Using the general form of $T_{0}$ given by (21), we can easily determine whether the eigenvalue 1 is multiple.

Proposition 18. If the solution of the equation with symbol $\mathbf{m}$ is continuous and $T_{0}$ has a multiple eigenvalue 1 , then $\mathbf{m}$ has a nontrivial generalized cycle $\mathbf{b}=\left\{b_{1}, \ldots, b_{n}\right\}$ such that

$$
\gamma_{\mathbf{b}}(\mathbf{m})=2^{k n} \quad \text { for some } k, 0 \leq k \leq r-1,
$$

where $r$ is the multiplicity of $\mathbf{b}$. The multiplicity of 1 in the spectrum of $T_{0}$ is equal to the total number of generalized cycles satisfying (59). The same number coincides with the dimension of the subspace $E \subset \mathbb{R}^{N}$ on which $T_{0}$ is the identity operator.

The simplest examples of the multiple eigenvalue 1 for $T_{0}$ are given by sparse equations (see $\S 11$ and also Examples 3 and 4). The proof of the following fact is elementary, and we leave it to the reader.

Corollary 13. Suppose the equation with a symbol $\mathbf{m}_{1}(z)$ has a continuous solution, and let $\mathbf{m}(z)=\mathbf{m}_{1}\left(z^{k}\right)$, where $k \geq 3$ is an odd integer. Then the matrix $T_{0}$ has a multiple eigenvalue 1. The dimension of the subspace $E$ is equal to the total number of cyclic sets that form the set of complex roots of unity of degree $k$.

Thus, if the eigenvalue 1 is not simple, then the operator $T_{0}$ has an invariant subspace $E, \operatorname{dim} E \geq 2$, on which $T_{0}$ is the identity. How do we find the initial vector $v_{0} \in E$ ? Since the solution $\varphi$ is unique, $v_{0}$ is also determined uniquely by the coefficients of the equation. This means that if another vector $v \in E$ is taken as the initial one, then the algorithm (58) does not converge to a continuous function. The vector $v_{0}$ is a unique element of $E$ possessing the following property: the joint spectral radius of the operators $T_{0}, T_{1}$ restricted to their smallest common invariant subspace containing the vector $\left(T_{1}-T_{0}\right) v_{0}$ is less than 1 . This result, which originates from [5], does not give, however, any practical method of finding $v_{0}$. This problem was studied in [5, 9, 10. Theorem[7provides a complete solution: $v_{0}$ is a unique eigenvector of $T_{0}$ that corresponds to the eigenvalue 1 and belongs to the subspace $\bar{V}$. If $\varphi \in C(\mathbb{R})$, then $\operatorname{dim}(\bar{V} \cap E)=1$. Using the explicit description of the space $\bar{V}$ given in Theorem 7 or in Proposition 11 , we find $\bar{V}$ and, after that, $v_{0}$. Furthermore, since $\rho\left(\left.T_{0}\right|_{V}\right)<1$, the vector $v_{0}$ is computed efficiently by the iterative approximation method.

If a solution of a refinement equation is not continuous, but only integrable, then its computation can be realized by a similar method: the vector $u_{0}=\int_{0}^{1} v(x) d x$ is an eigenvector of the operator $\frac{1}{2}\left(T_{0}+T_{1}\right)$ corresponding to the eigenvalue 1 . Having found $u_{0}$, we construct a piecewise linear function $u_{n}:[0,1] \rightarrow \mathbb{R}^{N}$ by the formula

$$
u_{n}(x)=\sum_{k=0}^{2^{n}-1} T_{d_{1}} \cdots T_{d_{n}} u_{0} \chi_{[0,1]}\left(2^{n} x-k\right), \quad 0 . d_{1} \cdots d_{n}=2^{-n} k .
$$

Thus, we approximate the solution $v(x)$ in the space $L_{p}[0,1]$. The deviation $\left\|u_{n}-v\right\|_{p}$ is estimated from above in terms of the regularity exponent $\alpha_{\varphi, p}$. We see that the construction of the solution is again reduced to finding an eigenvector (for the operator $\frac{1}{2}\left(T_{0}+T_{1}\right)$ in this case) corresponding to the eigenvalue 1 . Such a vector may fail to be unique. Complete analogs of Proposition 18 and Corollary 13 are valid for the operator $\frac{1}{2}\left(T_{0}+T_{1}\right)$. The problem is solved in the same way as above: on the space $\bar{V}$ 
the operator $\frac{1}{2}\left(T_{0}+T_{1}\right)$ possesses a unique eigenvector corresponding to the eigenvalue 1 , and it coincides with $u_{0}$ up to normalization.

Example 8. The equation with symbol $\mathbf{m}(z)=\frac{1}{4}\left(z^{6}+2 z^{3}+1\right)$ has a continuous solution. This symbol has a unique generalized cycle $\mathbf{b}=\left\{e^{2 \pi i / 3}, e^{4 \pi i / 3}\right\}$ of multiplicity $r_{1}=2$. Since $\gamma_{\mathbf{b}}(\mathbf{m})=1$, it follows that $\operatorname{dim} E=2$. Indeed,

$$
E=\left\{(0, t, 2 t, u, 2 t, t)^{T} \in \mathbb{R}^{6}, t, u \in \mathbb{R}\right\} .
$$

Thus, the vector $v_{0}$ lies in the two-dimensional subspace $E$. To compute $v_{0}$, first we find the subspace $\bar{V}$. We have

$$
C_{\mathbf{b}, 1}=\left(\begin{array}{cc}
1 & 0 \\
1 & 1 \\
1 & 1 \\
0 & 1
\end{array}\right) ; \quad C_{\mathbf{b}, 2}=\left(\begin{array}{cccc}
1 & 0 & 0 & 0 \\
1 & 1 & 0 & 0 \\
1 & 1 & 1 & 0 \\
0 & 1 & 1 & 1 \\
0 & 0 & 1 & 1 \\
0 & 0 & 0 & 1
\end{array}\right)
$$

Consequently, the subspace $\bar{V}$ is spanned by the columns of the matrix

$$
C_{\mathbf{b}, 2} \cdot C_{\mathbf{b}, 1}=\left(\begin{array}{cc}
1 & 0 \\
2 & 1 \\
3 & 2 \\
2 & 3 \\
1 & 2 \\
0 & 1
\end{array}\right)
$$

Thus, $\operatorname{dim} \bar{V}=2$. The intersection of the subspaces $\bar{V}$ and $E$ is one-dimensional, being spanned by the vector $(0,1,2,3,2,1)^{T}$. After normalization, we obtain $v_{0}=$ $\frac{1}{9}(0,1,2,3,2,1)^{T}$.

\section{$\S 16$. ThE MANIFOLD OF REFINEMENT EQUATIONS}

Theorem 2 guarantees the final clean symbol $\tilde{\mathbf{m}}(z)$ to be uniquely determined by the initial equation and to be independent of the order of iterations of the cleaning algorithm. Thus, all refinement equations are obtained from clean ones by adding symmetric zeros and cycles. Some basic properties of equations with clean symbols should be observed. Suppose a symbol $\tilde{\mathbf{m}}(z)$ is clean, $\tilde{\mathbf{m}}(1)=1$, and $\tilde{\varphi}, \tilde{T}_{i}$ are the corresponding refinable function and Toeplitz operators, respectively. Then the following is true:

1) The refinable function $\tilde{\varphi}$ is not integrable (see Lemma 7). Therefore, it is a singular distribution.

2) The operator $\frac{1}{2}\left(\tilde{T}_{0}+\tilde{T}_{1}\right)$ has the eigenvalue 1 . This implies that $\rho_{p}\left(\tilde{T}_{0}, \tilde{T}_{1}\right) \geq 1$ for any $p \in[1,+\infty]$.

Indeed, since $\sum_{k} c_{k}=2$, the sum of the entries in each column of the matrix $\frac{1}{2}\left(\tilde{T}_{0}+\tilde{T}_{1}\right)$ is equal to 1 .

3) The operators $\tilde{T}_{0}, \tilde{T}_{1}$ are both nondegenerate and have no common invariant subspaces (see Theorem 1).

We are going to classify all refinement equations generated by a given clean symbol $\tilde{\mathbf{m}}$. Suppose $z_{1}, \ldots, z_{q}$ are the roots of $\tilde{\mathbf{m}}(z)$ and $\mu_{1}, \ldots, \mu_{q}$ are their multiplicities. Thus, $\tilde{\mathbf{m}}(z)=R \prod_{k=1}^{q}\left(z-z_{k}\right)^{\mu_{k}}$, where $R=\prod_{k=1}^{q}\left(1-z_{k}\right)^{-\mu_{k}}$. Take an arbitrary family of cyclic sets $\mathbf{b}_{0}, \ldots, \mathbf{b}_{s}$, where $\mathbf{b}_{0}=\{1\}$, and the other cyclic sets are nontrivial and distinct (Figure 3). Let $r_{0}, \ldots, r_{s}$ be arbitrary natural numbers, and let $\mathcal{A}_{i}$ be arbitrary cut sets of multiplicities $r_{i}$ of the corresponding cyclic trees $\mathcal{T}_{\mathbf{b}_{i}}, i=0, \ldots, s$. Also, let $\mathcal{A}_{z_{k}}$ be 

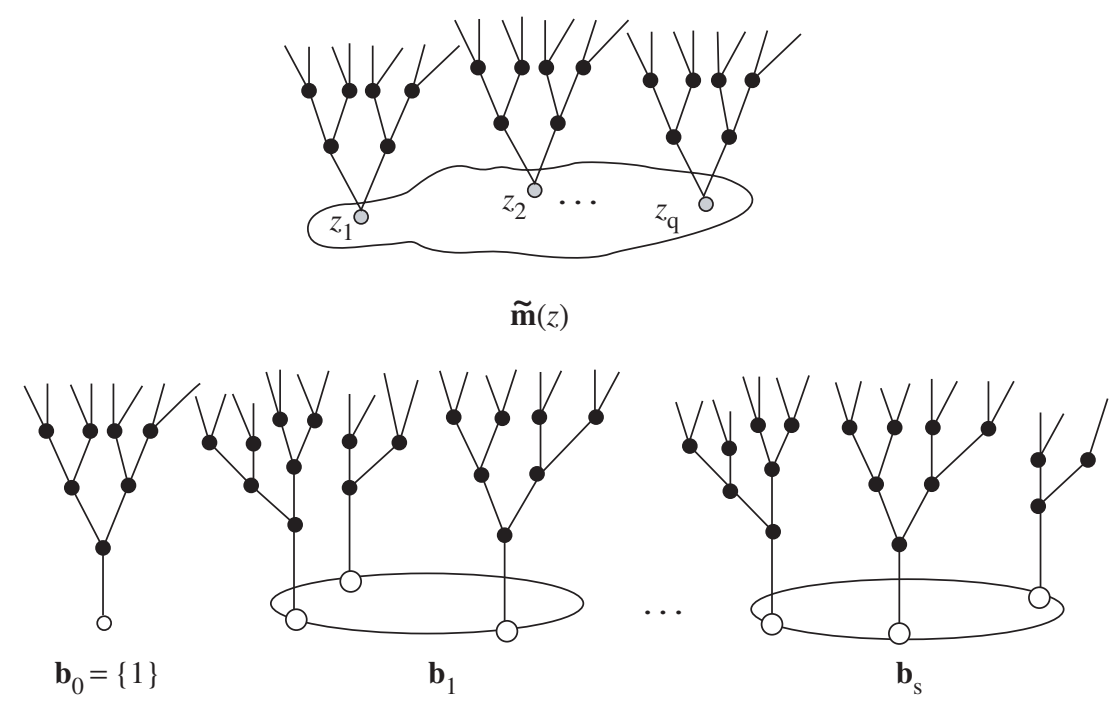

FIGURE 3 . The manifold of refinement equations with clean symbol $\tilde{\mathbf{m}}(z)$.

arbitrary cut sets of multiplicities $\mu_{k}$ of the trees $\mathcal{T}_{z_{k}}, k=1, \ldots, q$. Then all refinement equations generated by the clean symbol $\tilde{\mathbf{m}}$ are given by the formula

$$
\mathbf{m}(z)=2^{-r_{0}} R \cdot\left(\prod_{a \in \mathcal{A}_{i}, i=0, \ldots, s}(z-a)\right)\left(\prod_{a \in \mathcal{A}_{z_{k}}, k=1, \ldots, q}(z-a)\right) .
$$

The regularity of the corresponding refinable function $\varphi$ was determined in Theorem 9 , and the moduli of continuity in $L_{p}, p \in[1,+\infty]$, were found in Theorem 10, The rate of convergence of the cascade algorithm and the subdivision scheme for the symbol $\mathbf{m}$ are given by formula (53).

Thus, the problems concerning the solvability of refinement equations in given function spaces and computing the regularity exponents and the rates of convergence for cascade algorithms/subdivision schemes are all reduced to computing the spectral radii of the operators $\tilde{T}_{0}, \tilde{T}_{1}$ corresponding to the clean symbol $\tilde{\mathbf{m}}$. In the case of small degrees of $\tilde{\mathbf{m}}$, this latter problem can be solved explicitly; for large degrees it can be solved approximately by numerical algorithms for computing joint spectral radii.

The case where $\operatorname{deg} \tilde{\mathbf{m}}=0$. Refinable splines. In this case, the clean symbol is a constant, and the refinable function is a spline. Indeed, if $\mathbf{m}$ has neither symmetric zeros nor nontrivial cycles, then $\mathbf{m}(z)=\left(\frac{z+1}{2}\right)^{r}$ and $\tilde{\mathbf{m}}(z) \equiv 2^{-r}$, whence $\varphi(x)=B_{r-1}(x)$ is a cardinal $B$-spline. Otherwise, if there are either symmetric zeros or nontrivial cycles, then we apply the cleaning algorithm and obtain the corresponding $B$-spline. The transfer formulas (24) and (25) imply that the initial function $\varphi$ is a spline with integral nodes. For given $N$, there exist finitely many symbols of degree $N$ that generate refinable splines. They were classified in [16, 17]. In the second paper, the regularity exponents of refinable splines and the rate of convergence of the corresponding subdivision schemes were computed explicitly. The exponent $\alpha_{\varphi, p}$ of a refinable spline of order $r-1$ is equal to $r$ in the case of finite $p$ and to $r-1$ if $p=\infty$. Thus, this exponent is always an integer. Nevertheless, $\omega_{p}\left(\varphi^{(r-1)}, h\right) \asymp h$ (compare with Theorem 10). Surprisingly, the splines with integral nodes exhaust the set of piecewise smooth refinable functions (see [17]). 
The case where $\operatorname{deg} \tilde{\mathbf{m}}=1$. Two contractions on the line. The clean symbol is a degree 1 polynomial $\tilde{\mathbf{m}}(z)=2^{-r}(\lambda+(1-\lambda) z)$, where $\lambda$ is an arbitrary complex number different from 0 and $\frac{1}{2}$ (otherwise, the symbol can be reduced further to degree zero). In this case, $\tilde{T}_{0}$ and $\tilde{T}_{1}$ are the operators of multiplication on $\mathbb{R}$ by the numbers $2^{-r+1} \lambda$ and $2^{-r+1}(1-\lambda)$, respectively. Therefore,

$$
\rho_{p}=2^{-r+1-\frac{1}{p}}\left(|\lambda|^{p}+|1-\lambda|^{p}\right)^{1 / p}, \quad p<\infty ; \quad \rho_{\infty}=2^{-r+1} \max \{|\lambda|,|1-\lambda|\} .
$$

Now we can use Theorems 9, 10, and 13 to explicitly compute the regularity exponents of the refinable functions in $W_{p}^{l}$ and $C^{l}$ and the moduli of continuity and the rate of convergence of the corresponding cascade algorithms.

The case where $\operatorname{deg} \tilde{\mathbf{m}}=2$. The symbol is clean and of degree $2: \tilde{\mathbf{m}}(z)=2^{-r-1}\left(c_{2} z^{2}+\right.$ $\left.c_{1} z+c_{0}\right)$, where $c_{0}+c_{1}+c_{2}=2$ and $r$ is the multiplicity of the trivial generalized cycle for the initial symbol $\mathbf{m}$. If $c_{1}=1$ the symbol can be reduced further, and we return to one of the previous cases. Therefore, we assume that $c_{1} \neq 1$. The matrices $\tilde{T}_{i}$ are

$$
\tilde{T}_{0}=2^{-r}\left(\begin{array}{cc}
c_{0} & 0 \\
c_{2} & c_{1}
\end{array}\right) ; \quad \tilde{T}_{1}=2^{-r}\left(\begin{array}{cc}
c_{1} & c_{0} \\
0 & c_{2}
\end{array}\right) .
$$

The computation of $\rho_{p}$ becomes quite difficult. In contrast to the preceding examples, now the matrices do not commute and are not proportional to unitary matrices. We restrict ourselves to the case of real coefficients $c_{0}, c_{1}, c_{2}$ and focus on the joint spectral radius $\rho_{\infty}$ (responsible for the continuity of solutions).

Lemma 13. Suppose $c_{0}, c_{1}, c_{2}$ are real; then the matrices $\tilde{T}_{0}, \tilde{T}_{1}$ can be symmetrized simultaneously (i.e., there is a basis in which they are both symmetric) if and only if

$$
c_{0} c_{1} c_{2}\left(c_{1}-1\right) \geq 0 \text {. }
$$

Proof. The proof is elementary and we omit it.

The set of points $\left(c_{0}, c_{2}\right)$ satisfying (62) can easily be plotted on the coordinate plane. We have $c_{1}=2-c_{0}-c_{2}$. For every point of this set the computation of $\rho_{\infty}$ is simple, because $\rho_{\infty}=\max \left\{\rho\left(\tilde{T}_{0}\right), \rho\left(\tilde{T}_{1}\right)\right\}$ for symmetric matrices. Consequently, $\rho_{\infty}=$ $2^{-r} \max \left\{\left|c_{0}\right|\right.$, $\left.\left|c_{1}\right|,\left|c_{2}\right|\right\}$. Thus, we obtain the following statement.

Proposition 19. If $c_{0}, c_{1}, c_{2}$ are real and satisfy (62), then $\rho_{\infty}\left(\tilde{T}_{0}, \tilde{T}_{1}\right)=2^{-r} \max \left\{\left|c_{0}\right|\right.$, $\left.\left|c_{1}\right|,\left|c_{2}\right|\right\}$.

Note that the simultaneous symmetrization does not enable us to compute $\rho_{p}$ for all $p$ that easily. As to the pairs $\left(c_{0}, c_{2}\right)$ that do not satisfy (62), most likely, the joint spectral radius for them cannot be computed easily, even if we try to do this by looking over not two, but some small number of matrix products. The numerical results presented in [5] show that for some pairs $\left(c_{0}, c_{2}\right)$ all matrix products of lengths at most 20 do not give the exact value of the joint spectral radius. It is not even clear if for any point $\left(c_{0}, c_{2}\right)$ a finite exhaustion suffices.

Nevertheless, in the case of a symmetric symbol $\left(c_{0}=c_{2}\right)$ the problem has a relatively simple solution.

Proposition 20. If the coefficients $c_{0}, c_{1}, c_{2}$ are real and $c_{0}=c_{2}$, then for $c_{1} \in[0,1]$ we have $\rho_{\infty}=\sqrt{\rho\left(\tilde{T}_{0} \tilde{T}_{1}\right)}$, and for $c_{1} \notin[0,1]$ we have $\rho_{\infty}=\max \left\{\rho\left(\tilde{T}_{0}\right), \rho\left(\tilde{T}_{1}\right)\right\}$.

Proof. The proof is outlined in the Appendix. 
Thus, if either $c_{1} \leq 0$ or $c_{1} \geq 1$, then

$$
\rho_{\infty}=2^{-r} \max \left\{\left|c_{0}\right|,\left|c_{1}\right|,\left|c_{2}\right|\right\}=2^{-r} \max \left\{\left|c_{1}\right|,\left|1-\frac{c_{1}}{2}\right|\right\},
$$

and if $0 \leq c_{1} \leq 1$, then

$$
\rho_{\infty}=2^{-r-2} \max \left\{\left|2-c_{1}+\sqrt{4+12 c_{1}-7 c_{1}^{2}}\right|,\left|2-c_{1}-\sqrt{4+12 c_{1}-7 c_{1}^{2}}\right|\right\} .
$$

We do not know if there exist explicit formulas for the $p$-radii $\rho_{p}$ for finite $p$ under the assumptions of Proposition 20.

\section{APPENDIX}

Proof of Lemma 1. We establish the first equality in (5); the proof of the second is similar. The $k$ th coordinate of the vector $T_{0}^{*}\left[z^{2}\right]$ is

$$
\begin{aligned}
& \sum_{j=1}^{N} \bar{p}_{2 j-k-1}\left(z^{2}\right)^{j-1}=\sum_{j=1}^{N} \bar{p}_{2 j-k-1} z^{2 j-2} \\
& \quad=z^{k-1} \sum_{s \equiv k-1(\bmod 2)} \bar{p}_{s} z^{s}=\frac{1}{2}\left(z^{k-1} \overline{\mathbf{p}(\bar{z})}+(-z)^{k-1} \frac{1}{2} \overline{\mathbf{p}(-\bar{z})}\right),
\end{aligned}
$$

which concludes the proof.

Proof of Lemma 3. Assume that the set $A$ contains no symmetric pairs of numbers. Then for any $z_{1} \in B$ at least one of the numbers $\pm \sqrt{z_{1}}$ belongs to $B$. Denote this number by $z_{2}$. At least one of the numbers $\pm \sqrt{z_{2}}$ belongs to $B$; we denote it by $z_{3}$, etc. We obtain a sequence $\left\{z_{k}\right\} \subset B$. Since $B$ is finite, it follows that $z_{j}=z_{j+n}$ for some $j, n \in \mathbb{N}$. Then $z_{j}^{2^{n}}=z_{j}$, whence $z_{1}^{2^{n}}=z_{1}$. Thus, the set $\mathbf{b}=\left\{z_{1}, \ldots, z_{n}\right\} \subset B$ is cyclic. If $-\mathbf{b} \subset A$, then the proof is finished. Let $-z_{j} \notin A$ for some $z_{j} \in \mathbf{b}$. Then $-z_{j} \in B$, because $-z_{j} \in\left\{ \pm \sqrt{z_{j-1}}\right\} \subset A \cup B$. Arguing as above, we show that the number $-z_{j}$ belongs to some cyclic set $\mathbf{b}^{\prime} \subset B$. Observe that $z_{j-1}=\left(-z_{j}\right)^{2} \in \mathbf{b}^{\prime}$. Thus, the sets $\mathbf{b}^{\prime}$ and $\mathbf{b}$ have a nontrivial intersection, whence $\mathbf{b}^{\prime}=\mathbf{b}$. This is impossible because $-z_{j} \notin \mathbf{b}$. Therefore, $-\mathbf{b} \subset A$, which completes the proof.

Proof of Lemma 4. The existence of a root set has already been established. It remains to prove uniqueness. We apply induction on the number of elements of $Z$. Any root set is obtained from $Z$ by finitely many steps (transfers to the preceding level). Consider two sequences of steps leading to root sets $Z_{0}$ and $Z_{0}^{\prime}$, respectively. If these sequences begin with one and the same step, then after this step we obtain the same sets, and $Z_{0}=Z_{0}^{\prime}$ by the inductive hypothesis. Assume that they begin with different steps: the first sequence begins with a step $\left\{z_{0},-z_{0}\right\} \rightarrow z_{0}^{2}$, the second begins with some other step. Since all other steps do not involve the elements $z_{0},-z_{0}$, the second sequence contains the same step at some place; otherwise the set $Z_{0}^{\prime}$ would contain the two elements $\pm z_{0}$. We perform this step first, preserving the order of the other steps. This does not change the set $Z_{0}^{\prime}$. Then, as was already shown, $Z_{0}=Z_{0}^{\prime}$. This proves the lemma.

Proof of Lemma 5, a) Since $\mathbf{g}^{2}=\mathbf{g}$, we have

$$
\prod_{g \in \mathbf{g}}(z-g)=\prod_{g \in \mathbf{g}^{2}}(z-g)=\prod_{g \in \mathbf{g}}\left(z-g^{2}\right) .
$$


b) If $\mathbf{g}_{1} \neq \mathbf{g}_{2}$, then

$$
\begin{aligned}
& \prod_{g \in \mathbf{g}_{1}} P_{\mathbf{g}_{2}}(g)=\prod_{g_{1} \in \mathbf{g}_{1}, g_{2} \in \mathbf{g}_{2}}\left(\frac{g_{1}+g_{2}}{g_{2}}\right)=\prod_{g_{1} \in \mathbf{g}_{1}, g_{2} \in \mathbf{g}_{2}} \frac{g_{1}^{2}-g_{2}^{2}}{g_{2}\left(g_{1}-g_{2}\right)} \\
& =\left(\prod_{g_{1} \in \mathbf{g}_{1}, g_{2} \in \mathbf{g}_{2}}\left(g_{1}^{2}-g_{2}^{2}\right)\right):\left(\prod_{g_{1} \in \mathbf{g}_{1}, g_{2} \in \mathbf{g}_{2}}\left(g_{1}-g_{2}\right) g_{2}\right) \\
& \quad=\left(\prod_{g_{1} \in \mathbf{g}_{1}^{2}, g_{2} \in \mathbf{g}_{2}^{2}}\left(g_{1}-g_{2}\right)\right):\left(\prod_{g_{1} \in \mathbf{g}_{1}, g_{2} \in \mathbf{g}_{2}}\left(g_{1}-g_{2}\right) g_{2}\right)=\left(\prod_{g_{2} \in \mathbf{g}_{2}} g_{2}\right)^{-1}=1
\end{aligned}
$$

(as was shown before, the product of the elements of a cyclic set is 1 ). If $\mathbf{g}_{1}=\mathbf{g}_{2}$, then

$$
\begin{aligned}
& \prod_{g_{1} \in \mathbf{g}_{1}^{2}, g_{2} \in \mathbf{g}_{2}^{2}}\left(\frac{g_{1}+g_{2}}{g_{2}}\right)=\prod_{g_{1}=g_{2}}\left(\frac{g_{1}+g_{2}}{g_{2}}\right) \prod_{g_{1} \neq g_{2}}\left(\frac{g_{1}+g_{2}}{g_{2}}\right) \\
= & 2^{n} \prod_{g_{1} \neq g_{2}} \frac{g_{1}^{2}-g_{2}^{2}}{\left(g_{1}-g_{2}\right) g_{2}}=2^{n} \prod_{g_{1} \neq g_{2}} \frac{g_{1}^{2}-g_{2}^{2}}{\left(g_{1}-g_{2}\right)} \prod_{g_{2} \in \mathbf{g}_{2}} g_{2}^{-1}=2^{n} \prod_{g_{2} \in \mathbf{g}_{2}} g_{2}^{-1}=2^{n} .
\end{aligned}
$$

Proof of Proposition 10. Let $m(\xi)=\mathbf{m}\left(e^{-2 \pi i \xi}\right)$ be the symbol in the trigonometric form. Taking the Fourier transforms of both sides in (3) yields

$$
\left(a-e^{-4 \pi i \xi}\right) \widehat{\varphi}_{a}(2 \xi)=\left(a-e^{-4 \pi i \xi}\right) m_{a}(\xi) \widehat{\varphi}_{a}(\xi)=\left(a-e^{-2 \pi i \xi}\right) m(\xi) \widehat{\varphi}_{a}(\xi) .
$$

Then the function $a \varphi_{a}(x)-\varphi(x-1)$, which has the Fourier transform

$$
\left(a-e^{-2 \pi i \xi}\right) \widehat{\varphi}_{a}(\xi)
$$

satisfies the refinement equation with symbol $\mathbf{m}$. In the case of a nontrivial cycle the argument is similar:

$$
\begin{aligned}
Q_{\mathbf{b}}\left(e^{-4 \pi i \xi}\right) \widehat{\varphi}_{\mathbf{b}}(2 \xi) & =Q_{\mathbf{b}}\left(e^{-4 \pi i \xi}\right) \widehat{\varphi}_{\mathbf{b}}(\xi) m_{\mathbf{b}}(\xi)=Q_{\mathbf{b}}\left(e^{-2 \pi i \xi}\right) P_{\mathbf{b}}\left(e^{-2 \pi i \xi}\right) \widehat{\varphi}_{\mathbf{b}}(\xi) m_{\mathbf{b}}(\xi) \\
& =m(\xi) Q_{\mathbf{b}}\left(e^{-2 \pi i \xi}\right) \widehat{\varphi}_{\mathbf{b}}(\xi) .
\end{aligned}
$$

Therefore, the function $\sum_{k=0}^{n} q_{k} \varphi_{\mathbf{b}}(\cdot-k)$ with the Fourier transform $Q_{\mathbf{b}}\left(e^{-2 \pi i \xi}\right) \widehat{\varphi}_{\mathbf{b}}(\xi)$ satisfies the refinement equation with symbol $\mathbf{m}$. Finally, the function $\chi_{[0,1)}$ satisfies the refinement equation with symbol $\left(\frac{z+1}{2}\right)$, and the function $\varphi_{1}$ satisfies the refinement equation with symbol $2 \mathbf{m}_{1}$. Consequently, the convolution of these two functions satisfies the refinement equation with symbol $\left(\frac{z+1}{2}\right) 2 \mathbf{m}_{1}(z)=\mathbf{m}(z)$, which completes the proof.

The proof of Proposition 20 is based on the following lemma proved in 21].

Lemma 14. Let $B_{0}, B_{1}$ be arbitrary linear operators in $\mathbb{R}^{n}$, and let $\lambda$ be a positive real number. If there exists a convex body (compact convex set with nonempty interior) $\mathcal{M} \subset \mathbb{R}^{n}$ centrally symmetric with respect to the origin and such that $B_{i} \mathcal{M} \subset \lambda \mathcal{M}$, then $\rho_{\infty}\left(B_{0}, B_{1}\right) \leq \lambda$.

Now we prove Proposition 20. If either $c_{1} \leq 0$ or $c_{1} \geq 1$, then the coefficients satisfy (62). Then we apply Proposition 19 and conclude the proof. Suppose $0<c_{1}<1$. Clearly $\rho_{\infty} \geq \sqrt{\rho\left(\tilde{T}_{0} \tilde{T}_{1}\right)}$. To prove the reverse inequality, we use Lemma 14, Denote $y=\left(\sqrt{c_{0}^{2}+4 c_{1} c_{0}}-c_{0}\right) /\left(2 c_{1}\right), b=\left(1+y\left(\frac{c_{1}}{c_{0}}-1\right)\right) /\left(2-\frac{c_{1}}{c_{0}}\right)$. The desired convex body $\mathcal{M}$ is a convex hexagon with the vertices

$$
(1, y), \quad(-1,-y), \quad(y, 1), \quad(-y,-1), \quad(-b, b), \quad(b,-b) .
$$


It is easy to check that $\tilde{T}_{i} \mathcal{M} \subset \lambda \mathcal{M}$ for $i=0,1$, where $\lambda=\sqrt{\rho\left(\tilde{T}_{0} \tilde{T}_{1}\right)}=2^{-r-2}$. $\left|2-c_{1}+\sqrt{4+12 c_{1}-7 c_{1}^{2}}\right|$. Indeed, for $i=0$ we have $\tilde{T}_{0}( \pm 1, \pm y)=\lambda( \pm y, \pm 1), \tilde{T}_{0}( \pm y, \pm 1)$ $\in[\lambda( \pm y, \pm 1)], \lambda( \pm b, \mp b)], \tilde{T}_{0}( \pm b, \mp b) \in \lambda \mathcal{M}$. For $i=1$ the proof is the same.

This work was completed during the visit of the author to Catholic University of Louvain (Louvain-la-Neuve, Belgium). The author is grateful to the University for its hospitality.

\section{REFERENCES}

[1] G. Deslauriers and S. Dubuc, Symmetric iterative interpolation processes. Fractal approximation, Constr. Approx. 5 (1989), 49-68. MR.982724 (90c:65016)

[2] N. Dyn, J. A. Gregory, and D. Levin, Analysis of uniform binary subdivision schemes for curve design, Constr. Approx. 7 (1991), 127-147. MR1101059(92d:65027)

[3] A. S. Cavaretta, W. Dahmen, and C. A. Micchelli, Stationary subdivision, Mem. Amer. Math. Soc. 93 (1991), no. 453, 186 pp. MR.1079033 (92h:65017)

[4] I. Daubechies and J. Lagarias, Two-scale difference equations. II. Local regularity, infinite products of matrices and fractals, SIAM J. Math. Anal. 23 (1992), 1031-1079. MR1166574 (93g:39001)

[5] D. Colella and C. Heil, Characterization of scaling functions. I. Continuous solutions, SIAM J. Matrix Anal. Appl. 15 (1994), 496-518. MR1266600 (95f:26004)

[6] G. Strang, Eigenvalues of Toeplitz matrices with $1 \times 2$ blocks, Z. Angew. Math. Mech. 76 (1996), $37-39$.

[7] C. A. Cabrelli, C. Heil, and U. M. Molter, Polynomial reproduction by refinable functions, Advances in Wavelets (Hong Kong, 1997), Springer, Singapore, 1999, pp. 121-161. MR1688767|(2000g:42040)

[8] T. N. T. Goodman, C. A. Micchelli, G. Rodriguez, and S. Seatzu, Spectral factorization of Laurent polynomials, Adv. Comput. Math. 7 (1997), no. 4, 429-454. MR1470294(98e:65117)

[9] L. Berg and G. Plonka, Spectral properties of two-slanted matrices, Results Math. 35 (1999), no. 3-4, 201-215. MR.1694902 (2000c:15009)

[10] _ Some notes on two-scale difference equations, Functional Equations and Inequalities, Math. Appl., vol. 518, Kluwer Acad. Publ., Dordrecht, 2000, pp. 7-29. MR1792070 (2002f:39049)

[11] V. Protasov, Refinement equations with nonnegative coefficients, J. Fourier Anal. Appl. 6 (2000), no. 1, 55-78. MR.1756136 (2001i:42008)

[12] _ Asymptotics of the partition function, Mat. Sb. 191 (2000), no. 3, 65-98; English transl., Sb. Math. 191 (2000), no. 3-4, 381-414. MR1773255 (2001h:11134)

[13] _ Fractal curves and wavelets, Izv. Ross. Akad. Nauk Ser. Mat. 70 (2006), no. 5, 123-162. (Russian) MR2269711

[14] I. Daubechies, Ten lectures on wavelets, CBMS-NSF Regional Conf. Ser. in Appl. Math., vol. 61, SIAM, Philadelphia, 1992. MR1162107 (93e:42045)

[15] A. I. Kostrikin and Yu. I. Manin, Linear algebra and geometry, "Nauka", Moscow, 1986; English transl., Gordon and Breach Sci. Publ., New York, 1989. MR847051 (87f:00006) MR.1057342 (91h:00008)

[16] W. Lawton, S. L. Lee, and Z. Shen, Characterization of compactly supported refinable splines, Adv. Comput. Math. 3 (1995), no. 1-2, 137-145. MR1314906 (95m:41020)

[17] V. Yu. Protasov, Piecewise-smooth refinable functions, Algebra i Analiz 16 (2004), no. 5, 101-123; English transl., St. Petersburg Math. J. 16 (2005), no. 5, 821-835. MR2106669 (2005g:41023)

[18] C. A. Micchelli and H. Prautzsch, Uniform refinement of curves, Linear Algebra Appl. 114/115 (1989), 841-870. MR986909 (90k:65088)

[19] K. S. Lau and J. Wang, Characterization of $L_{p}$-solutions for two-scale dilation equations, SIAM J. Math. Anal. 26 (1995), 1018-1046. MR.1338372(96f:39004)

[20] G. Gripenberg, Computing the joint spectral radius, Linear Algebra Appl. 234 (1996), 43-60. MR:1368770 (97c:15043)

[21] V. Yu. Protasov, The joint spectral radius and invariant sets of linear operators, Fundam. Prikl. Mat. 2 (1996), no. 1, 205-231. (Russian) MR1789006 (2002d:47006)

[22] _ A generalized joint spectral radius. A geometric approach, Izv. Ross. Akad. Nauk Ser. Mat. 61 (1997), no. 5, 99-136; English transl., Izv. Math. 61 (1997), no. 5, 995-1030. MR.1486700 (99c:15041)

[23] D. X. Zhou, The p-norm joint spectral radius for even integers, Methods Appl. Anal. 5 (1998), 39-54. MR1631335 (99e:42054) 
[24] V. Protasov, A complete solution characterizing smooth refinable functions, SIAM J. Math. Anal. 31 (2000), no. 6, 1332-1350. MR1766557 (2001j:42033)

[25] I. Ya. Novikov, V. Yu. Protasov, and M. A. Skopina, Theory of wavelets, "Fizmatlit", Moscow, 2006, 612 pp. (Russian)

[26] M. Neamtu, Convergence of subdivision versus solvability of refinement equations, East J. Approx. 5 (1999), no. 2, 183-210. MR 1705396 (2000j:42054)

[27] R. Q. Jia, Subdivision schemes in $L_{p}$ spaces, Adv. Comput. Math. 3 (1995), no. 4, 309-341. MR 1339166 (96d:65028)

[28] Z. Wu, Convergence of subdivision schemes in $L_{p}$ spaces, Appl. Math. J. Chinese Univ. Ser. B 16 (2001), no. 2, 171-177. MR1833112 (2002c:42059)

[29] D. Chen and M. Han, Convergence of cascade algorithm for individual initial function and arbitrary refinement masks, Sci. China Ser. A 48 (2005), no. 3, 350-359. MR2158275 (2006b:42050)

[30] A. A. Melkman, Subdivision schemes with nonnegative masks converge always-unless they obviously cannot?, Ann. Numer. Math. 4 (1997), no. 1-4, 451-460. MR.1422696 (97i:41014)

Department of Mechanics and Mathematics, Moscow State University, 119992, Moscow, RUSSIA

E-mail address: vladimir_protassov@yahoo.com

Received 10/APR/2006

Translated by THE AUTHOR 